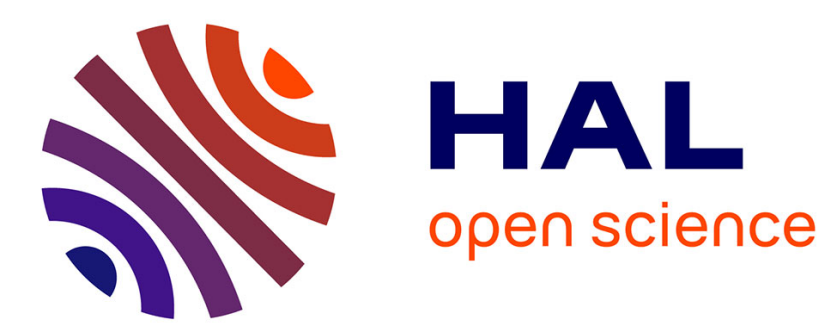

\title{
On stability condition for bifluid flows with surface tension : application to microfluidics
}

\author{
Cedric Galusinski, Paul Vigneaux
}

\section{To cite this version:}

Cedric Galusinski, Paul Vigneaux. On stability condition for bifluid flows with surface tension: application to microfluidics. Journal of Computational Physics, 2008, 227 (12), pp.6140-6164. 10.1016/j.jcp.2008.02.023 . hal-00274569v2

\section{HAL Id: hal-00274569 \\ https://hal.science/hal-00274569v2}

Submitted on 10 May 2008

HAL is a multi-disciplinary open access archive for the deposit and dissemination of scientific research documents, whether they are published or not. The documents may come from teaching and research institutions in France or abroad, or from public or private research centers.
L'archive ouverte pluridisciplinaire HAL, est destinée au dépôt et à la diffusion de documents scientifiques de niveau recherche, publiés ou non, émanant des établissements d'enseignement et de recherche français ou étrangers, des laboratoires publics ou privés. 


\title{
On stability condition for bifluid flows with surface tension : application to microfluidics
}

\author{
Cédric Galusinski ${ }^{\mathrm{a}}$, Paul Vigneaux ${ }^{\mathrm{b}, *}$, \\ a Université du Sud Toulon Var ; MC2 - INRIA Bordeaux; IMATH, Avenue de \\ l'Université - BP20132 La Garde Cedex, F-83957 France \\ ${ }^{\mathrm{b}}$ Université Bordeaux 1; MC2 - INRIA Bordeaux; CNRS ; UMR 5251 - IMB, \\ 351 Cours de la Libération, Talence, F-33405 France
}

\begin{abstract}
Models for incompressible immiscible bifluid flows with surface tension are here considered. Since Brackbill, Kothe and Zemach (J. Comput. Phys. 100, pp 335354, 1992) introduced the Continuum Surface Force (CSF) method, many methods involved in interface tracking or capturing are based on this reference work. Particularly, the surface tension term is discretized explicitly and therefore, a stability condition is induced on the computational time step. This constraint on the time step allows the containment of the amplification of capillary waves along the interface and puts more emphasis on the terms linked with the density in the Navier-Stokes equation (i. e. unsteady and inertia terms) rather than on the viscous terms. Indeed, the viscosity does not appear, as a parameter, in this stability condition.

We propose a new stability condition which takes into account all fluid characteristics (density and viscosity) and for which we present a theoretical estimation. We detail the analysis which is based on a perturbation study - with capillary wave - for which we use energy estimate on the induced perturbed velocity. We validate our analysis and algorithms with numerical simulations of microfluidic flows using a Level Set method, namely the exploration of different mixing dynamics inside microdroplets.
\end{abstract}

Key words: surface tension, curvature, stability condition, bifluid flows, incompressible Navier-Stokes, level set, cartesian finite-volumes, microfluidics, droplets

\footnotetext{
* Corresponding author. Current address : LAMA UMR 5127 CNRS ; Université de Savoie ; Campus Scientifique ; F-73376 Le Bourget-du-Lac ; France

Email address: Paul.Vigneaux@math.cnrs.fr (Paul Vigneaux).

URL: http://www . math.u-bordeaux.fr/ vigneaux/e-index.html (Paul Vigneaux).
} 


\section{Introduction}

Analysis and algorithms derived herein are the result of our interest in modelling and numerically simulate bifluid flows in microfluidics. Over the last decade, microfluidics has revolutionized our ability to manipulate and control flows in channels the width of a single human hair. The deeply affected fluid behaviour, due to preponderance of surface tension and viscosity, is used in applications ranging from biology and medicine to chemistry and materials processing. Among all applications of two fluids flows at low Reynolds numbers, we are more particularly interested in the use of microdroplets. Creation and transport of droplets in microchannels are extensively described in the literature both from theoretical and experimental point of view (e.g. see $[2,46,21])$. Making the most of surface tension effects, flows of two immiscible fluids in microdevices allow to create monodisperse emulsions where droplets of the same size move through microchannels networks and are used as microreactors to study very fast chemical kinetics (of the order of a millisecond $[45])$.

In this paper, we will thus consider models for immiscible bifluid flows with surface tension. A vast amount of numerical methods has been developed for modelling of such free surface flows. A standard classification first leads to distinguish Lagrangian, Eulerian or mixed Lagrangian-Eulerian methods regarding the modelling of the flow. In Lagrangian methods, a mesh element always contains the same fluid particles and thus computational mesh moves with the fluid. Conversely, Eulerian methods are based on a fixed mesh in which the fluid cross the computational cells. A second distinction lies in the modelling of the evolving interface which can be explicitly tracked along trajectories of fluid particles, leading to so-called interface tracking methods ; conversely, the interface can be implicitly tracked by embedding it in a globally defined field variable such as viscosity, density or volume fraction, leading to so-called interface capturing methods. Among the methods for simulating moving interface we find VOF [14,38], Level Set [31], front tracking [55,54], diffuse-interface $[1,4,23]$ and lattice Boltzmann $[7,12,16]$ methods, to name a few.

Among models for surface tension, the Continuum Surface Force (CSF) formulation introduced by Brackbill, Kothe and Zemach [5] has been widely and fruitfully used in the literature : e.g. see $[34,42]$ for VOF method or $[49,26]$ for Level Set method. The idea is to treat the surface tension as a body force in the momentum equation. This force, distributed within a transition zone, allows straightforward implementation of surface tension effect, even when topological changes occur. We note that numerous studies has been conducted to improve a drawback of this method, namely spurious currents 
(also known as parasitic currents) generated in the neighbourhood of the interface. These spurious currents are unphysical vortex-like velocities. First observed in Boltzmann interfacial methods, parasitic currents are also presented by Lafaurie et al. in [24] where they suggested the alternative Continuum Surface Stress (CSS) method. Then follow several approaches to tackle this problem $[35,32,53,34,41,52,26]$. Their key ideas in suppressing parasitic currents, usually mentioned in this literature, are $(i)$ improvement of curvature computation, ( $i i)$ achievement of discrete balance between surface tension and pressure gradient (iii) adaptive time integration scheme to tackle the stiffness induced by surface tension [25]. In addition, a singular and very promising work is developed by Jamet and coworkers [18]. It relies more on minimal energy consideration and can eliminate parasitic currents down to machine precision. Note, that this approach is applied in the context of diffuse interface and second gradient method [17].

In [5], as in many later approaches based on CSF method, the surface tension term is discretized explicitly and therefore, a stability condition is induced on the computational time step. This constraint on the time step allows the containment of the amplification of capillary waves along the interface. Furthermore most of the aforementioned methods use the stability condition derived in [5]. This stability condition puts more emphasis on the terms linked with the density in the Navier-Stokes equation (i. e. unsteady and inertia terms) rather than on the viscous terms. Indeed, the viscosity does not appear, as a parameter, in this stability condition. In this paper, we propose a new stability condition for which we present a theoretical estimation for flows with low and medium Reynolds numbers. This stability condition involves the fluid density as well as its viscosity. Besides, considering two regimes we can exhibit two stability conditions which are more restrictive and such that one of them is the condition proposed in [5] and the other is more suited for Stokes like flows. Numerical validation is done using a Level Set method.

Level Set methods have been applied with great success in a broad range of physical and image processing applications (see books [30] and [39]). The original formulation [31], together with tools of the Level Set technology such as (W)ENO schemes, TVD Runge-Kutta schemes [43,44,20,19] and PDE-based redistanciation are used here to achieve accurate simulation of surface tensiondriven flows. We note that numerical studies of flows at micrometer scale had already been conducted. In a series of papers, Yu, Sakai and Sethian [56-58] perform abundant numerical simulations of ink-jet printing for both Newtonian and viscoelastic fluids ; in these applications, the Reynolds number is rather high, namely 40 to 90 . Shapiro and Drikakis [40] developed specific methods for diffusion broadening in two- and three-dimensional microfluidic channels. De Menech performed simulation of droplet breakup in a microfluidic junction, with a phase field method [27]. Also concerning droplet formation, Renardy used very recently the VOF-PROST method to study the effects of 
confinement and inertia on the production of droplets [33]. In this paper, we will present numerical results for microflows with Reynolds number of order one or less, showing different mixing dynamics inside the microdroplets which are in good agreement with results reported in the literature.

The remainder of this paper is as follows. Section 2 is devoted to the presentation of mathematical models used for the simulation of bifluid flows with surface tension, adopting a CSF formulation. Section 3 then provides the description of the numerical resolution approach, discretizations and solvers. The main result of this paper concerning the new numerical stability condition is then derived in section 4 . The analysis is based on a perturbation study with capillary wave - for which we use energy estimate on the induced perturbed velocity. We will show numerically that a degenerate version - of this general stability condition - for Stokes like flows is better suited and discuss this point in more details. We will see that stability issue described herein has applications beyond the micrometre scale domain and, depending on fluids properties, can be applied to metre scale flow simulations. Finally, in section 5 , we present numerical results of microdroplets simulations.

\section{Concerned models for bifluid flows}

In this study, we consider flows of two immiscible fluids assumed to be viscous and Newtonian. We further assume that the flow is isothermal and fluids are incompressible and homogeneous. Densities and viscosities are thus constant within each fluid. The governing equations can then be expressed by the Navier-Stokes equation

$$
\rho\left(\frac{\partial \mathbf{u}}{\partial t}+\mathbf{u} \cdot \nabla \mathbf{u}\right)-\nabla \cdot(2 \eta D \mathbf{u})+\nabla p=\mathbf{F} \quad \forall(t, \mathbf{x}) \in \mathbb{R}^{+} \times \Omega
$$

together with the incompressibility condition :

$$
\nabla \cdot \mathbf{u}=0 \quad \forall(t, \mathbf{x}) \in \mathbb{R}^{+} \times \Omega,
$$

where $\Omega$ is the $2 \mathrm{D}$ (or $3 \mathrm{D}$ ) bounded fluid domain, $\mathbf{u}=(u, v)$ is the velocity field, $p$ the pressure, $\rho$ the density, $\eta$ the viscosity, $\mathbf{F}$ any body force (such as gravitational acceleration or surface tension, as we will describe in the following) and $D \mathbf{u}=\left(\nabla \mathbf{u}+\nabla^{T} \mathbf{u}\right) / 2$.

Bearing in mind that we will present some microfluidic applications at the end of this paper, we mention now that (1) reduces to Stokes equation when 
inertia influence can be neglected :

$$
\rho \frac{\partial \mathbf{u}}{\partial t}-\nabla \cdot(2 \eta D \mathbf{u})+\nabla p=\mathbf{F} \quad \forall(t, \mathbf{x}) \in \mathbb{R}^{+} \times \Omega
$$

Moving interfaces can be handled with the Level Set method - introduced by Osher and Sethian in [31] (see also [30] and [39]) - and we use here the approach of Sussman, Smereka and Osher [49] for incompressible two-phase flows. The interface between the two fluids is thus captured by advecting the Level Set function $\phi$ with the flow velocity $\mathbf{u}$ :

$$
\frac{\partial \phi}{\partial t}+\mathbf{u} . \nabla \phi=0 \quad \forall(t, \mathbf{x}) \in \mathbb{R}^{+} \times \Omega
$$

where $\phi$ has to be thought as a signed distance function. Affecting the subscript 1 and 2 to all quantities related respectively to fluid 1 or fluid 2, the Level Set function is, for instance, such that :

$$
\phi \begin{cases}<0 & \text { in fluid } 1 \\ =0 & \text { on the interface } \\ >0 & \text { in fluid } 2\end{cases}
$$

Hence (1) is a single fluid continuum model for the flow with variable density and viscosity given respectively by :

$$
\begin{aligned}
& \rho=\rho_{1}+\left(\rho_{2}-\rho_{1}\right) H(\phi) \\
& \eta=\eta_{1}+\left(\eta_{2}-\eta_{1}\right) H(\phi)
\end{aligned}
$$

where $H$ is the Heaviside function.

Being here dedicated to flows where surface tension is preponderant, we will assume in the following that gravitational acceleration is negligible and thus, the body force $\mathbf{F}$ is restricted to surface tension. In this sharp-interface approach, we further assume that surface tension is constant along the interface and we adopt the Level Set version of the CSF method to write the surface tension force $\mathbf{F}_{\sigma}$ as :

$$
\mathbf{F}_{\sigma}=\sigma \kappa \delta(\phi) \mathbf{n}
$$

where $\sigma$ is the surface tension coefficient, $\mathbf{n}$ is the unit normal to the interface, $\kappa$ is the curvature of the interface and $\delta(\phi)$ is the Dirac delta function localized on the interface. This formulation of the surface tension has been used by Unverdi 
and Tryggvason [55] and Brackbill, Kothe and Zemach [5]. This complete Level Set formulation with the Navier-Stokes equations for two-fluids flows was derived by Chang, Hou, Merriman and Osher [6] and later used in many other works (Sussman et al. [48,47], Olsson and Kreiss [29], Marchandise et al. [26], to new a few).

In a Level Set framework, the unit normal to the interface is classically obtained via $\phi$ :

$$
\mathbf{n}=\left.\frac{\nabla \phi}{|\nabla \phi|}\right|_{\phi=0}
$$

as well as the mean-curvature of the interface :

$$
\kappa=\left.\nabla \cdot\left(\frac{\nabla \phi}{|\nabla \phi|}\right)\right|_{\phi=0}
$$

\section{$3 \quad$ Numerical resolution approach}

In this section, we describe the general procedure, discretizations of the model and flow solver used to compute evolving interfaces for bifluid flows.

\subsection{General procedure}

In the following, we will consider geometry in two dimensions for ease of presentation. The 3D approach follows the same philosophy. The algorithm is as follows :

(1) Initialize a Level Set function $\phi$ to represent the interface and update physical quantities $\eta$ and $\rho$.

(2) Compute the unit normal $\mathbf{n}$ and the curvature $\kappa$.

(3) Solve the Navier-Stokes equation for $(\mathbf{u}, p)$.

(4) Update $\phi$ by solving the transport equation associated to $\mathbf{u}$.

(5) Eventually, apply redistanciation procedure on $\phi$, if needed.

(6) Iterate (2)-(5) for each step of the time discretization

Step (5) has been introduced in Level Set methods in order to improve mass conservation which is a drawback often mentioned in the literature. Mulder, Osher and Sethian [28] showed that taking $\phi$ as a signed distance function improve the accuracy of the method. Moreover, Chopp [8] went a step further 
introducing the concept of redistanciation : to achieve more accurate computation, $\phi$ should remain a signed distance function along iterations and thus should be periodically reinitialized ; here several approaches exist : on the one hand, reinitialization is applied at each time step, on the other hand, periodicity is strictly greater than one iteration (e.g. 10 iterations).

\subsection{Discretizations}

A first order discretization is used for evolving the equations in time. The superscripts $n$ and $n+1$ represent respectively the current and next time level. Following the algorithm presented in the last section, we have current $\mathbf{u}^{n}$ and $\phi^{n}$ which, by solving Navier-Stokes equation, gives $\left(\mathbf{u}^{n+1}, p^{n+1}\right)$; we can then solve transport equation for $\phi^{n+1}$. To sum up :

$$
\begin{aligned}
& \rho^{n}\left(\frac{\mathbf{u}^{n+1}-\mathbf{u}^{n}}{\Delta t}+\mathbf{u}^{n} \cdot \nabla \mathbf{u}^{n}\right)-\nabla \cdot\left(2 \eta^{n} D \mathbf{u}^{n+1}\right)+\nabla p^{n+1}=\sigma \kappa^{n} \delta\left(\phi^{n}\right) \mathbf{n} \\
& \nabla \cdot \mathbf{u}^{n+1}=0 \\
& \frac{\phi^{n+1}-\phi^{n}}{\Delta t}+\mathbf{u}^{n+1} \cdot \nabla \phi^{n}=0
\end{aligned}
$$

where $\Delta t$ is the computational time step. We would like to make several comments here. First, the surface tension term is discretized explicitly which implies a specific numerical stability condition as we will see in the following section. Second, one can also use higher order discretizations in time but this does not change the core result proposed in this paper. Finally, in our code, we can use TVD Runge-Kutta scheme in time of order 2 or 3 for the transport equation (following Shu and Osher [43]).

For spatial discretization of (11)-(12), we use a finite-volume method on a staggered grid as in the Marker and Cell (MAC) method of Harlow and Welch $[13]$.

Concerning the surface tension term $\sigma \kappa \delta(\phi) \mathbf{n}=\sigma \nabla \cdot \mathbf{n} \nabla H(\phi)$, we use a mollified Heaviside function on few cells (e.g. 3) and a central scheme to approximate the curvature $\nabla \cdot \mathbf{n}=\nabla \cdot \frac{\nabla \phi}{|\nabla \phi|}$. Note that, for a slightly improvement of

numerical results, we prefer to approximate the curvature $\nabla \cdot \frac{\nabla \tilde{\phi}}{|\nabla \tilde{\phi}|}$ where $\tilde{\phi}$ is a five-point average of $\phi$.

The transport equation (13) is discretized with a WENO5 scheme [19].

For the redistanciation of the function $\phi$, we use a reinitialization equation 
which is discretized by the method of Russo and Smereka [36].

\subsection{Flow solver}

In order to solve (11)-(12) for $\left(\mathbf{u}^{n+1}, p^{n+1}\right)$, we use an augmented Lagrangian method (see e.g. $[50,9,11]$ ).

This algorithm consists in solving Navier-Stokes with an iterative method in order to converge towards a solution which satisfies the incompressibility constraint. To this end, we denote in a natural way $\left(\mathbf{u}_{k+1}, p_{k+1}\right)$ and $\left(\mathbf{u}_{k}, p_{k}\right)$ the variables of this iterative process. We proceed as follows :

(1) Initialize $\left(\mathbf{u}_{0}, p_{0}\right)$ (e.g. solving Stokes equation)

(2) Solve the following linear system for $\mathbf{u}_{k+1}$ :

$$
\begin{aligned}
& \frac{\rho}{\Delta t} \mathbf{u}_{k+1}-\nabla \cdot\left(2 \eta D \mathbf{u}_{k+1}\right)+\theta_{1} \nabla\left(\nabla \cdot \mathbf{u}_{k+1}\right)= \\
& \frac{\rho}{\Delta t} \mathbf{u}^{n}-\rho \mathbf{u}^{n} \cdot \nabla \mathbf{u}^{n}+\sigma \kappa^{n} \delta\left(\phi^{n}\right) \mathbf{n}-\nabla p_{k}
\end{aligned}
$$

(3) Update the pressure $p_{k+1}$ via

$$
p_{k+1}=p_{k}-\theta_{2} \nabla \cdot \mathbf{u}_{k+1}
$$

(4) Iterate (2)-(3) until convergence

(e.g. when $\left|p_{k+1}-p_{k}\right|<\zeta$ or $\left|\nabla \cdot \mathbf{u}_{k+1}\right|<\zeta$ )

(5) Finally, assign $\left(\mathbf{u}^{n+1}, p^{n+1}\right)=\left(\mathbf{u}_{k+1}, p_{k+1}\right)$

where $\theta_{1}$ and $\theta_{2}$ are numerical coefficients of the augmented Lagrangian and $\zeta$ is the desired convergence criteria. In our computations, we take $\theta_{1}=\theta_{2}=1$. Remark that the initialization step (1) can be done as follows, depending on your current status in the global evolution computation :

- if the initial physical time step of the simulation must be computed, and one does not have any "natural" initial guess neither for $\left(\mathbf{u}^{0}, p^{0}\right)$ and thus nor for $\left(\mathbf{u}_{0}, p_{0}\right)$, one can compute the solution of the stationary Stokes equation. To this end, it suffices to apply the above algorithm with any initial $\left(\mathbf{u}_{0}, p_{0}\right)$ $\left(\right.$ e.g. $\left.\left(\mathbf{u}_{0}, p_{0}\right)=(1 ; 1)\right)$ and imposing $\rho=0$. At convergence, obtained solution is an ad hoc candidate $\left(\mathbf{u}^{0}, p^{0}\right)$ for initializing unsteady Navier-Stokes computation

- if several iterations are already computed, one could simply assign $\left(\mathbf{u}_{0}, p_{0}\right)$ $=\left(\mathbf{u}^{n}, p^{n}\right)$

In microfluidic applications of this paper, where a Stokes model is used for the flow, this augmented Lagrangian algorithm converges in 4 or 5 iterations 
to the solution such that the vanishing-divergence constraint is verified at the order of the divergence approximation, say at the second order.

\section{Stability condition and the role of surface tension}

Formulation (11)-(12)-(13) classically induces a numerical stability condition on the time step. First, the time step must obey the CFL condition due to the convective terms of Navier-Stokes and transport equation. Second, the explicit discretization of the surface tension term induces another restriction on the time step. This constraint on the time step allows the containment of the amplification of capillary waves along the interface.

Most of methods based on the CSF formulation use the surface tension-induced stability condition derived in the seminal work of Brackbill et al. [5] and other derivations also leads to similar conditions (e.g. see [22]).

In this section, we derive a new stability condition induced by surface tension for flows with low and medium Reynolds numbers.

\subsection{Stability analysis}

Proposition 1 Assume that (1) (2) is discretized in time by an explicit discretization of the surface tension term and that (4) is discretized by a stable explicit scheme. Then, for sufficiently small Reynolds numbers, a numerical scheme, associated to such a time discretization and all space discretizations, is stable under the condition

$$
\begin{aligned}
& \Delta t \leq \min \left(\Delta t_{c}, \Delta t_{\sigma}\right), \text { with } \\
& \Delta t_{c}=c_{0}\|\mathbf{u}\|_{L^{\infty}(\Omega)}^{-1} \Delta x \text { and } \\
& \Delta t_{\sigma}=\Delta t_{\sigma}(\rho, \eta)=\frac{1}{2}\left(c_{2} \frac{\eta}{\sigma} \Delta x+\sqrt{\left(c_{2} \frac{\eta}{\sigma} \Delta x\right)^{2}+4 c_{1} \frac{\rho}{\sigma} \Delta x^{3}}\right)
\end{aligned}
$$

where $\Delta t$ is the time step, $\Delta x$ is the space step of the discretization, and $c_{0}$, $c_{1}, c_{2}$ do not depend on the physical and discretization data of the problem.

Remark 2 In this proposition, the restriction on the Reynolds number holds only because of the nonlinear term in Navier-Stokes equations. This restriction corresponds to an assumption of laminar flows. 
Remark 3 Note that, as shown in (18), the time step $\Delta t_{\sigma}$ depends on the density and the viscosity. The capillary time step derived in [5] verifies

$$
\Delta t_{B K Z} \sim \sqrt{\frac{\rho}{\sigma} \Delta x^{3}} \sim \Delta t_{\sigma}(\rho, 0)
$$

The capillary time step related to Stokes equation (when neglecting inertia phenomena)

$$
\Delta t_{S T K} \sim \frac{\eta}{\sigma} \Delta x \sim \Delta t_{\sigma}(0, \eta)
$$

Finally, we remark that the capillary time step $\Delta t_{\sigma}$ is the less restrictive, since

$$
\Delta t_{\sigma} \geq \Delta t_{S T K} \quad \text { and } \quad \Delta t_{\sigma} \geq \Delta t_{B K Z}
$$

Proposition 1 then shows numerical stability under the well known condition

$$
\Delta t \leq \min \left(\Delta t_{c}, \Delta t_{B K Z}\right)
$$

Moreover, this proposition also shows numerical stability under the condition

$$
\Delta t \leq \min \left(\Delta t_{c}, \Delta t_{S T K}\right)
$$

We will particularly focus on numerical validation of (23) in following section 4.2 and then discuss and compare all these conditions in section 4.3.

We note that the following derivation is not, in a strict sense, a mathematical proof since two relevant physical assumptions on the Navier-Stokes solutions are introduced step by step in the derivation. Actually, these assumptions allow to complete the mathematical derivation of inequality verified by a "capillary velocity" (which will be precised below) and are thus useful for numerical analysis of the time step constraint. Apart from these two relevant assumptions the whole derivation consists in rigorous mathematical analysis.

\section{DERIVATION.}

First, convection terms imply the constraint $\Delta t \leq c_{0}\|\mathbf{u}\|_{L^{\infty}(\Omega)}^{-1} \Delta x$ which is the classical CFL condition where $c_{0}$ depends on the choice of the scheme to discretize the transport equation (4).

Second, the condition involving $\Delta t \leq \Delta t_{\sigma}$ avoids the oscillation phenomena of the interface due to surface tension and this condition is the main objective of the present derivation. Let us begin by outlining how we proceed : 


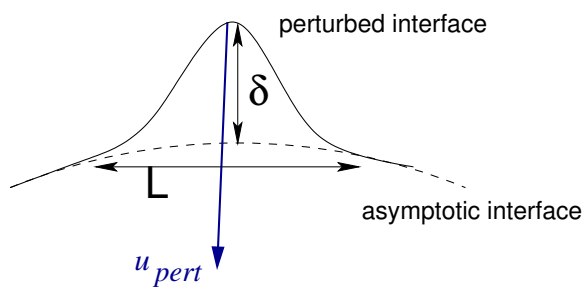

Fig. 1. A perturbed interface (of amplitude $\delta$ and wavelength $L$ ) and induced velocity

(1) When (11)-(12)-(13) is solved numerically, some small consistent numerical errors lead to some equally small perturbations of the interface shape which in turn induce a perturbation velocity, also called capillary velocity since it is due to surface tension (see Figure 1).

(2) In order for the explicit discretization (11) to be stable with respect to surface tension influence, one needs to choose a sufficiently small time step so that the displacement (during the time step) of the interface is smaller than the size $\delta$ of the perturbation of the interface (see Figure 1), i.e. :

$$
\Delta t_{\sigma} \leq \frac{\delta}{\|\mathbf{w}\|}
$$

where $\|\mathbf{w}\|$ is the norm of the perturbed velocity (which will be defined in the following) induced by surface tension.

(3) In order to find the stability condition, we thus need to find a refined bound on velocity with respect to the perturbation of the interface : this is the key point of the analysis and the new contribution compared to previous heuristics.

In order to gauge the extent of this perturbed velocity, we perform the analysis on the continuous problem instead of the discrete problem. For that, we assume that the numerical scheme approximates consistently the continuous problem.

We consider a smooth interface $\Gamma_{0}(t)$, at a time $t$, and assume it is parametrized as :

$$
\Gamma_{0}(t)=\left\{\left(x_{\Gamma_{0}}(s) ; y_{\Gamma_{0}}(s)\right) \in \mathbb{R}^{2} / s \in[-1 ; 1)\right\}
$$

Let then $f$ be a $C^{2}$ function with $\operatorname{supp}(f) \subset[-1 ; 1]$ such that $\|f\|_{C^{0}}=1$ and $\|f\|_{C^{2}}=O(1)$. We consider the following perturbation of $\Gamma_{0}(t)$ :

$$
\Gamma(t)=\left\{\left(x_{\Gamma_{0}}(s) ; y_{\Gamma_{0}}(s)+\delta f\left(\frac{s}{L}\right)\right) \in \mathbb{R}^{2} / s \in[-1 ; 1)\right\}
$$

where $\delta$ and $L$ are respectively the amplitude and the wavelength of a small 
perturbation. We will see that we are concerned with small wavelength $L$, which generates high curvatures variations and, in turn, high local velocities. The limitation on time step is such that the numerical scheme has to predict an interface $\Gamma(t+\Delta t)$ with a smaller perturbation than the one of $\Gamma(t)$. The same analysis can then be performed when considering a perturbation of the velocity instead of a perturbation of the interface.

Let us recall that in the Level Set framework, a regularization of Dirac delta function $(\nabla H(\phi))$ is obtained by considering $H_{\varepsilon}$ instead of $H$, where $H_{\varepsilon}$ is a smooth increasing function and approximates the Heaviside function as $\varepsilon$ goes to zero, $\operatorname{supp}_{\varepsilon}^{\prime} \subset(-\varepsilon ; \varepsilon),\left\|H_{\varepsilon}^{\prime}\right\|_{\infty} \leq \frac{2}{\varepsilon}$.

Let us define $\kappa_{0}(x, y)($ resp. $\kappa(x, y))$, the curvature of $\Gamma_{0}$ (resp. $\left.\Gamma\right)$ at a point $(x, y) \in \mathbb{R}^{2}$. Both curvatures $\kappa_{0}$ and $\kappa$ are useful in an $\varepsilon$-neighbourhood of $\Gamma_{0}$ and $\Gamma$ when the Dirac delta function on the interface is $\varepsilon$-regularized. Nevertheless, in order to simplify the analysis and to estimate the source term, the curvatures are extended on the full domain in such a way that

$$
\left\|\nabla \kappa_{0}\right\|_{L^{\infty}(\Omega)}=\left\|\partial_{s} \kappa_{0}\right\|_{L^{\infty}\left(\Gamma_{0}\right)},\|\nabla \kappa\|_{L^{\infty}(\Omega)}=\left\|\partial_{s} \kappa\right\|_{L^{\infty}(\Gamma)}
$$

where $\partial_{s}$ denotes the tangential derivative along $\Gamma$ or $\Gamma_{0}$.

Let us recall the so-called standard pressure shift that we use in order to reformulate source term (8) of Navier-Stokes equation (1), namely $\sigma \kappa \nabla H_{\varepsilon}(\phi)$. By the chain rule we have

$$
\kappa \nabla H_{\varepsilon}(\phi)=\nabla\left(\kappa H_{\varepsilon}(\phi)\right)-(\nabla \kappa) H_{\varepsilon}(\phi) .
$$

Thus

$$
\rho\left(\partial_{t} \mathbf{u}+\mathbf{u} . \nabla \mathbf{u}\right)-\nabla \cdot(2 \eta D \mathbf{u})+\nabla p=\sigma \kappa \nabla H_{\varepsilon}(\phi)
$$

can be rewritten as ( $\sigma$ being constant):

$$
\rho\left(\partial_{t} \mathbf{u}+\mathbf{u} \cdot \nabla \mathbf{u}\right)-\nabla \cdot(2 \eta D \mathbf{u})+\nabla p=\nabla\left(\sigma \kappa H_{\varepsilon}(\phi)\right)-\sigma(\nabla \kappa) H_{\varepsilon}(\phi)
$$

where pure gradient term $\nabla\left(\sigma \kappa H_{\varepsilon}(\phi)\right)$ can then be included to pressure term so that :

$$
\rho\left(\partial_{t} \mathbf{u}+\mathbf{u} . \nabla \mathbf{u}\right)-\nabla \cdot(2 \eta D \mathbf{u})+\nabla\left(p-\sigma \kappa H_{\varepsilon}(\phi)\right)=-\sigma(\nabla \kappa) H_{\varepsilon}(\phi)
$$

Finally, we made the following change of variable (keeping the same notation) $p-\sigma \kappa H_{\varepsilon}(\phi) \rightarrow p$ also known as pressure shift. The reformulated Navier-Stokes 
equation becomes :

$$
\rho\left(\partial_{t} \mathbf{u}+\mathbf{u} . \nabla \mathbf{u}\right)-\nabla \cdot(2 \eta D \mathbf{u})+\nabla p=-\sigma(\nabla \kappa) H_{\varepsilon}(\phi)
$$

We can now begin to find a bound on the perturbed velocity. To do so, we write equation (32) associated to both interfaces $\Gamma_{0}$ and $\Gamma$. We denote $\mathbf{u}$ the velocity field associated to the interface $\Gamma_{0}$ and verifying

$$
\left\{\begin{array}{l}
\rho\left(\partial_{t} \mathbf{u}+\mathbf{u} . \nabla \mathbf{u}\right)-\nabla \cdot(2 \eta D \mathbf{u})+\nabla p_{0}=-\sigma\left(\nabla \kappa_{0}\right) H_{\varepsilon}\left(\phi_{0}\right) \\
\nabla \cdot(\mathbf{u})=0
\end{array}\right.
$$

and $\mathbf{v}$ the velocity field associated to the interface $\Gamma$ and verifying

$$
\left\{\begin{array}{l}
\rho\left(\partial_{t} \mathbf{v}+\mathbf{v} \cdot \nabla \mathbf{v}\right)-\nabla \cdot(2 \eta D \mathbf{v})+\nabla p=-\sigma(\nabla \kappa) H_{\varepsilon}(\phi) \\
\nabla \cdot(\mathbf{v})=0
\end{array}\right.
$$

We then denote $\mathbf{w}=\mathbf{v}-\mathbf{u}$ and take the difference (34)-(33) to obtain (assuming $\rho$ and $\eta$ are constant and denoting $\left.q=p-p_{0}\right)$ :

$$
\begin{aligned}
& \nabla .(\mathbf{w})=0 \\
& \rho \partial_{t} \mathbf{w}-\nabla \cdot(2 \eta D \mathbf{w})+\rho \mathbf{v} \cdot \nabla \mathbf{w}+\rho \mathbf{w} \cdot \nabla \mathbf{u}+\nabla q= \\
& -\sigma\left[\nabla\left(\kappa-\kappa_{0}\right)\right] H_{\varepsilon}(\phi)-\sigma\left[\nabla \kappa_{0}\right]\left[H_{\varepsilon}(\phi)-H_{\varepsilon}\left(\phi_{0}\right)\right]
\end{aligned}
$$

One can show that right-hand side terms verify following inequalities :

$$
\begin{aligned}
& \left\|\left[\nabla\left(\kappa-\kappa_{0}\right)\right] H_{\varepsilon}(\phi)\right\|_{L^{\infty}(\Omega)} \leq c \frac{\delta}{L^{3}} \\
& \left\|\left[\nabla \kappa_{0}\right]\left[H_{\varepsilon}(\phi)-H_{\varepsilon}\left(\phi_{0}\right)\right]\right\|_{L^{\infty}(\Omega)} \leq\left\|\partial_{s} \kappa_{0}\right\|_{L^{\infty}\left(\Gamma_{0}\right)} \frac{c \delta}{\varepsilon},
\end{aligned}
$$

the source term of (36), denoted $g$ below, is then bounded at time $t=0$, in $L^{\infty}\left(\mathbb{R}^{2}\right)$ norm by :

$$
\|g(0)\|_{L^{\infty}(\Omega)} \leq c \sigma\left(\frac{\delta}{L^{3}}+\left\|\partial_{s} \kappa_{0}\right\|_{L^{\infty}\left(\Gamma_{0}\right)} \frac{\delta}{\varepsilon}\right)
$$

where $c$ does not depend on $\delta, L, \varepsilon$ and $\sigma$. We note that the inequality involving $\varepsilon$ is not optimal when $\varepsilon$ goes to zero, but we will see that it is sufficient for the analysis with $\varepsilon \sim \Delta x$. 
We will now perform a so called $L^{2}$ energy estimate of (36) [51]. It consists in multiplying equation (36) by $\mathbf{w}$ and integrating resulting equation over $\Omega$. Taking into account that $\mathbf{w}$ is divergence free, the pressure gradient term vanishes ; then by use of definition of $L^{2}(\Omega)$-norm, Green theorem and CauchySchwarz inequality, it reads :

$$
\frac{\rho}{2} \frac{d}{d t}\|\mathbf{w}\|_{L^{2}(\Omega)}^{2}+\eta\|\nabla \mathbf{w}\|_{L^{2}(\Omega)}^{2} \leq\|g\|_{L^{2}(\Omega)}\|\mathbf{w}\|_{L^{2}(\Omega)}+\rho\|\nabla \mathbf{u}\|_{L^{\infty}(\Omega)}\|\mathbf{w}\|_{L^{2}(\Omega)}^{2}(40)
$$

After this mathematically rigorous analysis, we decide to introduce

Assumption 4 which is the first assumption of this derivation, namely

$$
\|\nabla \mathbf{w}\|_{L^{2}(\Omega)} \sim \frac{1}{L}\|\mathbf{w}\|_{L^{2}(\Omega)}
$$

i.e. a source term induces a velocity perturbation which is essentially of the same wavelength $L$.

Note that this assumption is numerically verified as it can be seen for instance on Figure 4 (where vortex size is of same order of the interface perturbation's wavelength). Furthermore, a rigorous justification of (41) is probably a work of its own, even for the Stokes equation.

Thanks to (41), there exists a constant $C$ such that

$$
\frac{\rho}{2} \frac{d}{d t}\|\mathbf{w}\|_{L^{2}(\Omega)}^{2}+\left(\frac{C \eta}{L^{2}}-\rho\|\nabla \mathbf{u}\|_{L^{\infty}(\Omega)}\right)\|\mathbf{w}\|_{L^{2}(\Omega)}^{2} \leq \frac{L^{2}}{C \eta}\|g\|_{L^{2}(\Omega)}^{2} .
$$

Note that, because (41) is not an equality, we only know that $C$ is of order one but its value is not known exactly. This plays a role in the fact that it will not be possible to predict an exact value of $c_{2}$. This will be discussed later.

To continue the derivation, we now introduce

Assumption 5 which is second and last assumption of this derivation, namely

$$
\rho\|\nabla \mathbf{u}\|_{L^{\infty}(\Omega)} \leq \frac{C \eta}{2 L^{2}}
$$

which is true for sufficiently low Reynolds numbers.

Then, the end of the derivation is completely mathematically rigorous and relies on standard mathematical analysis tools for partial differential equations. By plugging (43) into (42) and using Gronwall's lemma we get : 


$$
\begin{aligned}
\|\mathbf{w}(t)\|_{L^{2}(\Omega)}^{2} \leq & \|\mathbf{w}(0)\|_{L^{2}(\Omega)}^{2} \exp \left(-\frac{C \eta}{\rho L^{2}} t\right) \\
& +\left(1-\exp \left(-\frac{C \eta}{\rho L^{2}} t\right)\right) \frac{L^{4}}{C^{2} \eta^{2}} \sup _{s \in(0, t)}\|g(s)\|_{L^{2}(\Omega)}^{2} .
\end{aligned}
$$

If we consider that the initial velocity is not perturbed, $\mathbf{w}(0)=0$ and the source term $g$ is maximal for $t=0$, finally

$$
\|\mathbf{w}(t)\|_{L^{2}(\Omega)} \leq\left(1-\exp \left(-\frac{C \eta}{\rho L^{2}} t\right)\right) \frac{L^{2}}{C \eta}\|g(0)\|_{L^{2}(\Omega)}, \quad \forall t>0 .
$$

Using again the profile of the solution (as in assumption (41)) and the surface tension term, we have

$$
\begin{aligned}
& \|\mathbf{w}\|_{L^{2}(\Omega)} \sim L^{\prime}\|\mathbf{w}\|_{L^{\infty}(\Omega)} \\
& \|g\|_{L^{2}(\Omega)} \sim L^{\prime}\|g\|_{L^{\infty}(\Omega)}
\end{aligned}
$$

Assumptions (46) and inequality (45) yield

$$
\|\mathbf{w}(t)\|_{L^{\infty}(\Omega)} \leq\left(1-\exp \left(-\frac{C \eta}{\rho L^{2}} t\right)\right) \frac{L^{2}}{C \eta}\|g(0)\|_{L^{\infty}(\Omega)}, \quad \forall t>0 .
$$

Considering a time discretization of (36) with an implicit discretization of the diffusive term and an explicit discretization of the source term, discrete analog of (47) on a time step $\Delta t$ is

$$
\|\mathbf{w}(\Delta t)\|_{L^{\infty}(\Omega)} \leq \frac{\Delta t}{\rho \frac{L^{2}}{C \eta}+\Delta t} \frac{L^{2}}{C \eta}\|g(0)\|_{L^{\infty}(\Omega)}, \quad \forall t>0 .
$$

As mentioned in the previous outline (on page 11), we can now, with (24), determine an inequality verified by the capillary time step $\Delta t_{\sigma}$. If the displacement of the interface is larger than $2 \delta$, perturbations are amplified and oscillates. Finally, with (24)-(39)-(48) combined, we can write the stability condition by saying that the oscillations are removed if

$$
\Delta t_{\sigma}=\frac{\delta}{\left\|\mathbf{w}\left(\Delta t_{\sigma}\right)\right\|_{L^{\infty}(\Omega)}} \leq c \frac{\rho \frac{L^{2}}{C \eta}+\Delta t_{\sigma}}{\Delta t_{\sigma}} \frac{\eta}{\sigma} \frac{L}{1+\left\|\partial_{s} \kappa_{0}\right\|_{L^{\infty}\left(\Gamma_{0}\right)} \frac{L^{3}}{\varepsilon}} .
$$

As the wavelength $L$ is upper bounded, this condition is restrictive for the smaller admissible wavelength in the numerical process. We are then concerned 
with $L \sim \Delta x$, which gives

$$
\Delta t_{\sigma}^{2} \leq c_{2} \frac{\eta}{\sigma} \Delta x \Delta t_{\sigma}+c_{1} \frac{\rho}{\sigma} \Delta x^{3}
$$

with $c_{1}, c_{2}$ two positive constants independent of physical and numerical parameters. We finally obtain (18) which completes the derivation in the case where we consider that initial velocity is not perturbed.

If we consider a perturbation (with a given wavelength $L$ ) of the initial velocity, $\mathbf{w}(0) \neq 0$, instead of a perturbation of the interface, the interface is deformed with the same wavelength. The same analysis can be performed starting from (44). The maximal value of $g$ is reached for a positive time, corresponding to a maximal value of the amplitude of the deformation on the interface.

The goal of the next two subsections is, first, to validate from a numerical point of view the stability condition (23) and, second, to discuss features linked with this derivation and compare it with previous heuristics described in the literature. Even if the condition induced by (18) is the less restrictive one, condition (23) is a pertinent sufficient stability condition when considering flows driven by capillary instability. This point will also be discussed in the following subsection.

\subsection{Numerical confirmation of the stability condition}

We now present numerical simulations which validate the stability condition

$$
\Delta t_{S T K}=c_{2} \frac{\eta}{\sigma} \Delta x
$$

This time step is smaller than $\Delta t_{\sigma}$ but is close when the inertia phenomena are small. Microfluidics is a typical example of such flows where surface tension is preponderant, and thus, unconfined droplets have a near circular shape which translates in straight channels, when the flow velocity is low. We will use this framework in order to simulate these kind of physically stable interfaces and will show that constant $c_{2}$ exhibited in our derivation exists. Namely, there exists a threshold value for $c_{2}$ such that if $c_{2}$ is chosen under this threshold (resp. above) the simulation becomes numerically stable (resp. unstable).

We perform numerical simulations in two dimensions taking the parameters in such a way we simulate microflows. Namely, we consider a rectangular channel with a section of $120 \cdot 10^{-6} \mathrm{~m}$. The maximum of injection velocity is $9.10^{-2}$ 
$\mathrm{m} / \mathrm{s}$. Viscosity and density are equal in both fluid : $\eta_{1}=\eta_{2}=2 \cdot 10^{-2}$ and $\rho_{1}=\rho_{2}=10^{-8}$. Surface tension is $\sigma=3 \cdot 10^{-2}$. Discretization in space is such that there are 36 cells in the section and 80 cells in the direction of the channel.

Note that with these parameters, we take, on purpose, a vanishing density in such a way that stability condition (51) is (i) in the order $10^{5}$ times greater than condition proposed by Brackbill et al. and (ii) more restrictive than CFL condition. The latter is classically expected, contrary to (i). This will be further discussed in the next subsection.

We point out the quality of numerical simulations through viewing the velocity field in the frame of moving interface. We consider droplets moving in a straight channel, where the droplet's shape has to converge to an asymptotic shape so that the velocity field in the drop frame of reference is tangential to the interface. Details on how to exhibit the drop frame of reference are given in Appendix A.

As a first test case, we take as initial condition an interface which is ellipsoidal such that it converges to a near circular droplet shape (with diameter equal to $2 / 3$ of channel section) under the mentioned flow conditions. This initial state is shown on the left of Figure 2. An asymptotic stable shape is obtained in finite time and shown on the right of Figure 2. In this test, $c_{2}=8$. The numerical simulation remains stable for all computational time and one can observe that the asymptotic shape is reached since streamlines in the droplet's frame of reference are tangent to the interface.
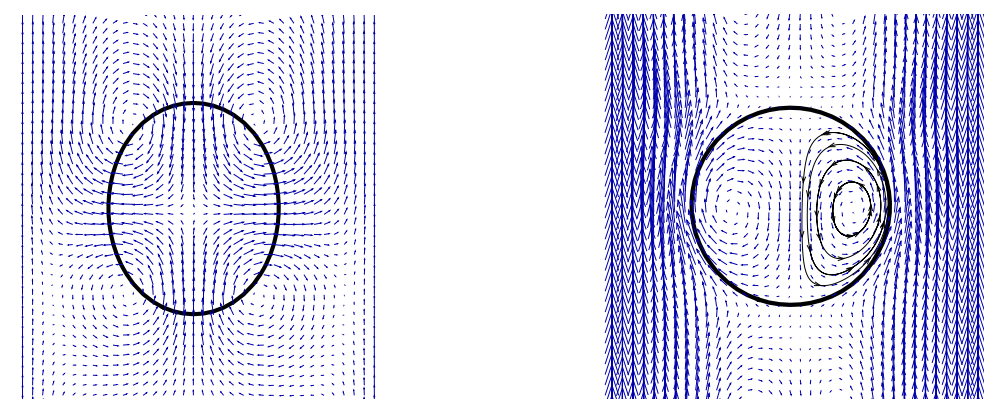

Fig. 2. Left : initial state of simulation. Right : asymptotic state of the droplet. The interface is represented by a thick black line and the velocity field in the droplet's frame of reference is represented by blue arrows. Note that the arrows' scale of the right is 10 times the one on the left. On the right, streamlines are added with black thin arrowed lines and show that the asymptotic state has been reached. Here $c_{2}=$ 8.

Such snapshot is always obtained for values of $c_{2}$ such that $: c_{2} \leq 8$; this is the threshold above which simulations become unstable. As a matter of fact, 
if we run the same simulation with $c_{2}=9$, the interface first converges toward the asymptotic shape and then becomes unstable. On the left of Figure 3, we see the same initial state presented on the left of Figure 2 except that we use the arrow scale of the right of Figure 2 ; this allows to have an idea of the scaling change. On the right of Figure 3, the snapshot at the same time of the right of Figure 2 is shown and clearly exhibits the numerical instabilities due to the violation of our condition. When looking at the evolution of this interface, we see that it oscillates around the asymptotic with an amplitude growing with time, leading to inconsistent results and eventually a break of the simulation. Such parasitic currents do not have to be confused with the ones discussed in the introduction which are of greater size and which do not diminish with mesh refinement.
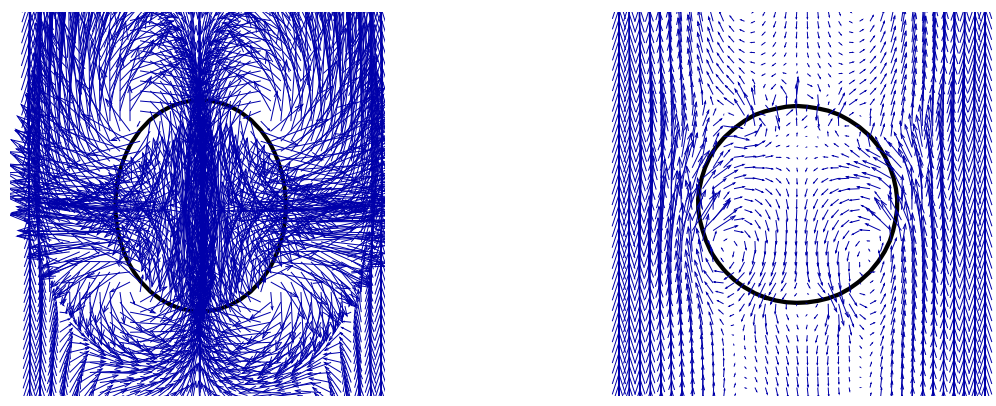

Fig. 3. Left : initial state of simulation. Right : asymptotic state of the droplet. The interface is represented by a thick black line and the velocity field in the droplet's frame of reference is represented by blue arrows. Note that the arrows' scale is the same on the left and on the right, as well as on the right of Figure 2. There, we can clearly see that the simulation is unstable with parasitic currents near the interface. The asymptotic state is never reached and oscillations of both velocity field and interface grow along time and can induce a break of the simulation. Here $c_{2}=9$.

Note that we also perform same simulations which show that this threshold value $c_{2}=8$ is unchanged under mesh refinement, which numerically proves the independence of $c_{2}$ with respect to the mesh size, as shown in our derivation. In the same manner, this threshold is independent of $\eta$ and $\sigma$. Furthermore, if $\rho$ is increased this value of $c_{2}$ also leads to numerically stable simulations as it is predicted by the analysis. For large $\rho$, note that the stability condition induced by (20) is not optimal and can be relaxed to the one induced by (18).

With our computation of curvature, the critical value to develop instabilities is around $c_{2}=8$. For such a value, instabilities are very long to develop or to decrease (for $c_{2}$ slightly under 8). The good choice for the constant is then $c_{2}$ $=4$ according to our analysis. As a matter of fact, the value $c_{2}=8$ is close to the value ensuring symmetric oscillations, along the time, of the interface when oscillations have a wavelength of the same order as the mesh size. Then, the constant $c_{2}=4$ (half of 8 ) is the biggest value ensuring no oscillations of 
perturbed interface. For $c_{2}$ between 4 and 8, oscillations of small wavelength occurs but are damped. Note that the constant $c_{2}$ is dependent on the way of computation of the numerical curvature ; this will be discussed in the following subsection.

Then, we describe two other tests which show the independence of the threshold with respect to the wavelength of the perturbation.

The second test is the same as the first one except that we change the initial interface at $t=0$ by taking a circle (whose diameter is equal to $2 / 3$ of the channel section) perturbed with a cosine of amplitude $\Delta x$ and wavelength $\lambda_{5}=2 \pi r_{c} / 5$, where $r_{c}$ is the radius of the circle (cf. snapshot on the left of Figure 4). As shown on Figure 4, with $c_{2}=4$, the initial interface converges to previously mentioned asymptotic interface and remains stable by translating in the channel for all simulation time.
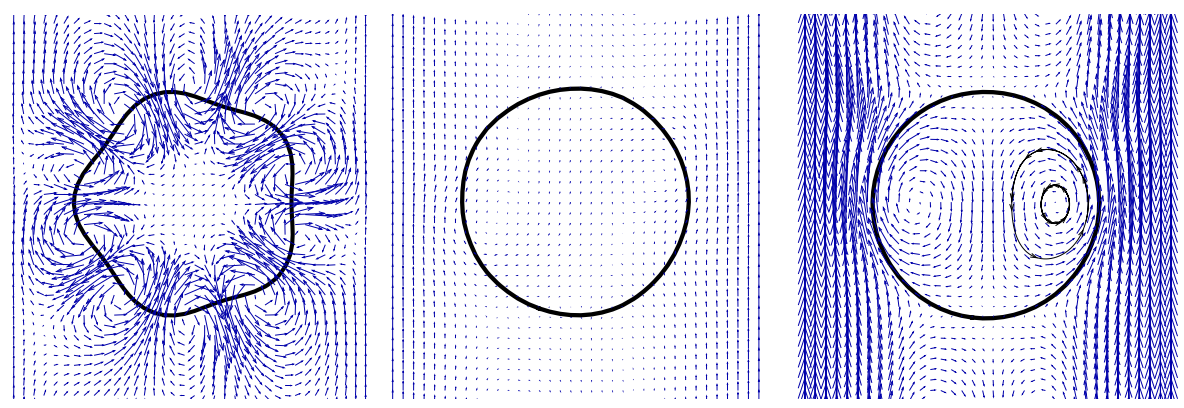

Fig. 4. Evolution of an initial interface (on the left) perturbed with a cosine of amplitude $\Delta x$ and wavelength $\lambda_{5}$, with $c_{2}=4$ (To be compared with Figure 5). The code is able to converge to the asymptotic shape which translates in the channel. The interface is represented by a thick black line and the velocity field in the droplet's frame of reference is represented by blue arrows. Note that the arrows' scale of the right is 10 times the one on the left and middle pictures. On the right, streamlines are added with black thin arrowed lines and show that the asymptotic state is reached.

On the contrary, if $c_{2}=10$ is chosen, the same initial interface first converges to the circular asymptotic shape but then begins to destabilize with spurious velocities : capillary instabilities grows with time and are not controlled, as it can be seen on Figure 5 where all snapshots are taken at same times as in Figure 4 .

The third test case is the same as the second one except for a change in the wavelength of the perturbation by taking $\lambda_{20}=2 \pi r_{c} / 20$. Again we observe 

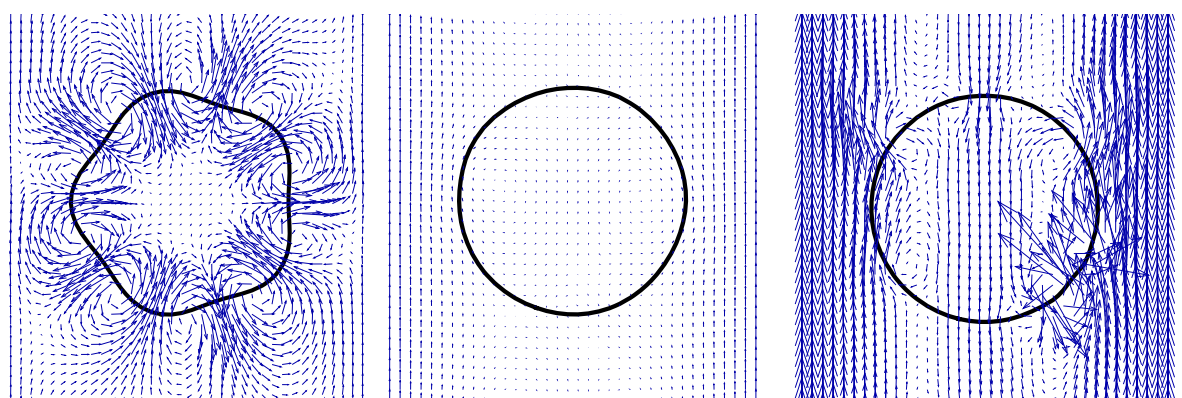

Fig. 5. Evolution of an initial interface (on the left) perturbed with a cosine of amplitude $\Delta x$ and wavelength $\lambda_{5}$, with $c_{2}=10$ (To be compared with Figure 4). The code does not converge to the asymptotic shape. The interface is represented by a thick black line and the velocity field in droplet's frame of reference is represented by blue arrows. Note that the arrows' scale of the right is 10 times the one on the left and middle pictures. Spurious velocities, induced by violation of stability condition linked to surface tension, appear on the right.

the same behaviour when running the code with $c_{2}=4$ and $c_{2}=10$. Figure 6 shows, on the left, the initial condition and associated velocity field, and on the middle and the right, convergence of interface to asymptotic shape : the computation is stable with $c_{2}=4$.
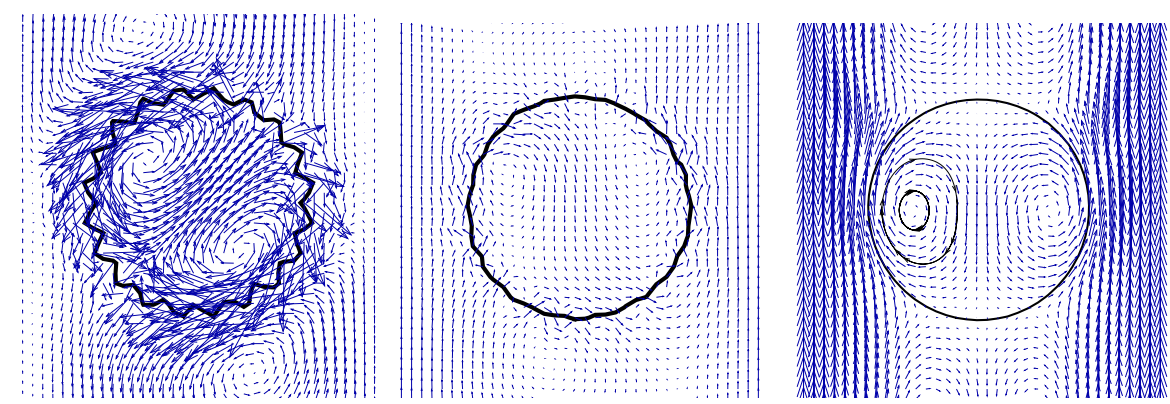

Fig. 6. Evolution of an initial interface (on the left) perturbed with a cosine of amplitude $\Delta x$ and wavelength $\lambda_{20}$, with $c_{2}=4$ (To be compared with Figure 7). The code is able to converge to the asymptotic shape which translates in the channel. The interface is represented by a thick black line and the velocity field in droplet's frame of reference is represented by blue arrows. Note that the arrows' scale of the right is 10 times the one on the left and middle pictures. On the right, streamlines are added with black thin arrowed lines and show asymptotic state is reached.

Conversely, Figure 7 shows that if $c_{2}=10$ is chosen, simulation always exhibits spurious currents originating from the interface : the code is not able to damp the oscillations induced by surface tension.

On Figures 5 and 7 where at initial condition long $\left(\lambda_{5}=4 \lambda_{20}\right)$ and moderate $\left(\lambda_{20}=3 \Delta x\right)$ wavelength are introduced, we see that code is able to damp 

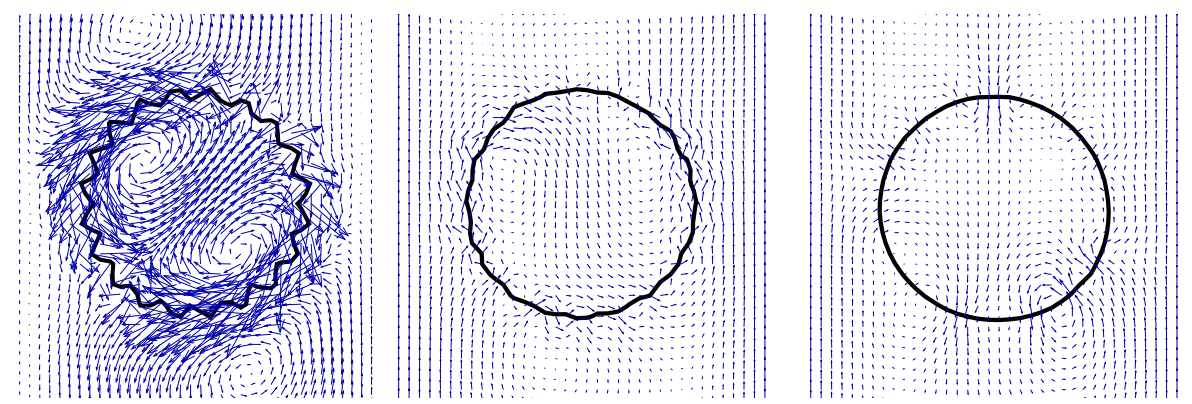

Fig. 7. Evolution of an initial interface (on the left) perturbed with a cosine of amplitude $\Delta x$ and wavelength $\lambda_{20}$, with $c_{2}=10$ (To be compared with Figure 6). The code does not converge to the asymptotic shape. The interface is represented by a thick black line and the velocity field in droplet's frame of reference is represented by blue arrows. Note that the arrows' scale is the same for the three snapshots.

associated oscillations to a certain level (see snapshot in the middle of Figures 5 and 7 ) but then, the resulting perturbations - which have a wavelength of order $\Delta x$-develop and for $c_{2}=10$, they grow and are not controlled by the code (see snapshot on the right of Figure 5 and 7 ). Note furthermore that the minimal wavelength which can be computed by the code is theoretically $\sqrt{2} \Delta x$. And by the way, we see that with $c_{2}=10$ the code is able to damp oscillations with wavelength greater than $3 \Delta x$ but can not damp smaller wavelengths of order $\Delta x$. Conversely, with an appropriate value of $c_{2}$ (i.e. $c_{2} \leq 8$ ), the code is able to damp all oscillations of the interface induced by surface tension.

It must be noted that for both kind of perturbation (wavelength $\lambda_{5}$ and $\lambda_{20}$ ) the threshold value for $c_{2}$ is still $c_{2}=8$. Thus, and again as a forgone conclusion, thanks to the previous derivation, $c_{2}$ is independent of the wavelength of the perturbation.

\subsection{Discussion and remarks}

We would like to make several comments here about the stability condition derived above and compare it to previous capillary stability conditions.

The stability constraint (20) can be very restrictive in applications where the velocity of the flow is very low compared to $\frac{\sigma}{\eta}$. As mention by Brackbill et al. [5], it would be interesting to achieve an implicit discretization of the surface tension term, in order to remove this constraint. Due to the highly non-linear coupling induced by $\phi$ in (1)-(2)-(4), implicit treatment of surface tension is not an easy task. A step in this direction was recently performed by Hysing in [15] where he proposed a semi-implicit discretization of the surface tension term (see also preceding works of Bänsch [3]). 
The present derivation of the stability condition shows that if the curvature is regularized (for instance, via an interpolation procedure as it is the case in several methods) then the capillary time step $\Delta t_{\sigma}$ is increased. As a matter of fact, perturbations of the interface with a small wavelength $L$ and a small amplitude $\delta$ are modified by a smoothing effect leading to a smaller amplitude $\delta$ and a larger wavelength $L$. Following the derivation of Proposition 1, the perturbed velocity $\mathbf{w}$ is lower. This thus leads to a relaxation of the time step constraint. For this reason, the constant $c_{2}$ is not universal since it depends on how curvature is computed. To summarize on quantification of $c_{2}$, one has to figure out that taking into account present derivation :

- $c_{2}$ is of order one (this has also been shown numerically) for a computed curvature assumed to lead to the worst case ;

- thus, if numerical method leads to smoothed curvature, $c_{2}$ can be of an order greater than one ;

- and, because of assumption 4 which is not an equality, it is not possible to give an exact value of $c_{2}$ by algebra and taking into account formula used to compute curvature.

In the next two subsubsections, we point out previous derivations of capillary stability conditions and compare them with respect to linked flow characteristics.

\subsubsection{Comparison with previous heuristics}

To our knowledge, the first derivation of a stability condition induced by surface tension forces is given in [5]. Different from the one derived here, this capillary stability condition is :

$$
\Delta t_{B K Z} \sim \sqrt{\frac{\rho}{\sigma}} \Delta x^{3 / 2}
$$

The derivation of (52) puts more emphasis on the terms linked with the density in the Navier-Stokes equation (i. e. unsteady and inertia terms) rather than on the viscous terms. Indeed, the viscosity does not appear, as a parameter, in this stability condition.

The same stability condition as (52) is given in [22] with a heuristic based on an estimate of the capillary velocity. Nevertheless the estimate of this velocity takes into account an equation on velocity reduced to

$$
\rho \frac{\partial \mathbf{u}}{\partial t} \sim \sigma \kappa \delta(\phi) \mathbf{n}
$$


leading to the following estimate (argued on dimensional grounds) of discrete capillary velocity

$$
u_{B K Z} \sim \frac{\sigma}{\rho} \frac{\Delta t_{\sigma}}{\Delta x^{2}}
$$

Note that in the present paper, full Navier-Stokes equations are taken into account and we perform as far as we can rigorous mathematical analysis which is then completed by two relevant physical assumptions (for which rigorous proof is arguably beyond the scope of this paper) and allows to derive a different capillary velocity :

$$
u_{\sigma} \sim \frac{\sigma}{\rho \Delta x^{2}+c \Delta t_{\sigma} \eta} \Delta t_{\sigma}
$$

This reduces to a stronger velocity for Stokes equation $(\rho=0)$

$$
u_{S T K} \sim \frac{\sigma}{\eta}
$$

Then, estimations of capillary velocity are plugged in a CFL-like stability condition, i.e.

$$
\Delta t_{*}=\frac{\Delta x}{u_{*}}
$$

Consequently, three stability conditions induced by surface tension can be obtained :

- (52) if velocity (54) is used

- or (18) if velocity (55) is used.

- or (20) if velocity (56) is used.

For low Reynolds, the time step (20) is close to (18) and has been validated in section 4.2, we discuss below a comparison of (52) and (20) with respect to flow characteristics.

\subsubsection{Time steps associated to various flow regimes}

In order to compare two previously mentioned capillary time steps, let us recall that $\Delta t_{c}$ is the classical CFL time step, $\Delta t_{B K Z}$ is the time step derived in [5], $\Delta t_{S T K}$ the time step derived here with $\rho=0$, and let us associate the corresponding velocities: 


$$
\begin{aligned}
\Delta t_{c} & \sim \frac{1}{\|\mathbf{u}\|_{L^{\infty}(\Omega)}} \Delta x \\
\Delta t_{B K Z} & \sim \sqrt{\frac{\rho}{\sigma}} \Delta x^{3 / 2}:=\frac{1}{u_{B K Z}} \Delta x, \\
\Delta t_{S T K} & \sim \frac{\eta}{\sigma} \Delta x:=\frac{1}{u_{S T K}} \Delta x .
\end{aligned}
$$

Remark that capillary velocities verify $u_{S T K}=\frac{\sigma}{\eta}$ and $u_{B K Z}=\sqrt{\frac{N}{R e}} \sqrt{\|\mathbf{u}\|_{L^{\infty}(\Omega)} u_{S T K}}$, where $N$ is the number of mesh cells in the direction of the characteristic length (e.g. channel's diameter).

When the capillary velocity $u_{S T K}$ is high compared to the flow velocities (due to low injection), the stability condition induced by time step (60) is restrictive, but when the Reynolds number is small,

$$
R e \ll N \frac{\|\mathbf{u}\|_{L^{\infty}(\Omega)}}{u_{S T K}},
$$

the time step (59) is even smaller. In the previous paragraph, we have seen that our stability condition suffices for stable simulations and is close to (18).

For sufficiently high Reynolds,

$$
R e \gg N \frac{\|\mathbf{u}\|_{L^{\infty}(\Omega)}}{u_{S T K}},
$$

the time step (59) is less restrictive than the time step (60). In such regimes, inertia phenomena are preponderant, the stability condition (60) (optimal only for low Reynolds) has then to be replaced by (18) which is close to (59) when viscosity vanishes. Nevertheless, for such regimes $(R e \rightarrow \infty)$, it is not clear that we are concerned with capillary instabilities because of turbulent flows inducing physical instabilities of the interface. It becomes difficult to distinguish numerical and physical instabilities of the interface.

To conclude this section, we return to the well known time step (19),

$$
\Delta t_{B K Z}=\sqrt{c_{1} \frac{\rho}{\sigma} \Delta x^{3}}
$$


where $c_{1}$ can be numerically calibrated on the system

$$
\begin{aligned}
& \rho \frac{\partial \mathbf{u}}{\partial t}+\nabla p=\sigma \kappa \delta(\phi) \mathbf{n} \\
& \nabla \cdot \mathbf{u}=0 \\
& \frac{\partial \phi}{\partial t}+\mathbf{u} \cdot \nabla \phi=0
\end{aligned}
$$

The constants $c_{2}$ and $c_{1}$ being known, it is then possible to compute the less restrictive time step $\Delta t_{\sigma}$ induced by (18). Nevertheless, since

$$
\max \left(\Delta t_{S T K}, \Delta t_{B K Z}\right) \leq \Delta t_{\sigma} \leq \frac{1+\sqrt{5}}{2} \max \left(\Delta t_{S T K}, \Delta t_{B K Z}\right)
$$

for all regimes, $\Delta t_{\sigma}$ is always equal to

$$
\Delta t_{\sigma}=\alpha \max \left(\Delta t_{S T K}, \Delta t_{B K Z}\right)
$$

with $1 \leq \alpha \leq 1.62$. Consequently, the two time steps $\Delta t_{S T K}, \Delta t_{B K Z}$ are the two main quantities to determine a pertinent stability condition by taking the maximum of these two values.

For microfluidic applications, small dimensions lead to low inertia phenomena, the maximal value of $\Delta t_{S T K}$ and $\Delta t_{B K Z}$ is $\Delta t_{S T K}$.

\section{Microfluidic applications}

Due to preponderant effect of surface tension, flows of two immiscible fluids in microdevices allow to create monodisperse emulsions where droplets of the same size moves through microchannels networks and are used as microreactors to study very fast chemical kinetics.

In this section, we show numerical simulations of such microdroplets obtained with the Level Set method described previously together with the new stability condition. As we are interested in the mixing dynamics inside microdroplets, we essentially present velocity fields and streamlines in the drop frame of reference, for moving interface with stabilized shape (see Appendix A). We note that in microflows, due to confinement, 3D effects have to be considered in order to obtain a full description of the flow. Nonetheless, we will see that 2D simulations are a first step allowing to have a qualitative description of mixing dynamics which is in good agreement with physical experiments. In all the following Figures presenting numerical simulations, droplets move from top to bottom. 
We begin by presenting an unconfined droplet in a microchannel on Figure 8 . The droplet (in black) is plotted together with the global velocity field and a perfect circle (in red) in order to show the accuracy of the method and the influence of surface tension : even if the curvature is a small perturbation of a circle, the velocity field is noticeably modified. On Figure 9, we also present the

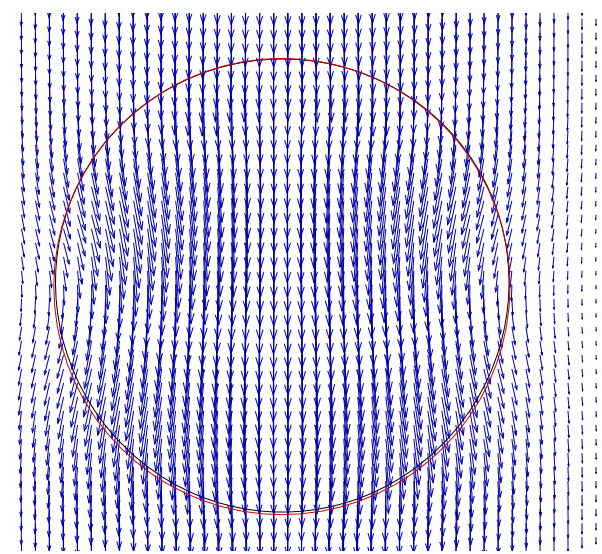

Fig. 8. Unconfined microdroplet (black line) together with the associated global velocity field (blue arrows) and a perfect circle (red line).

associated velocity field in the drop frame of reference and some streamlines which reveal the mixing dynamics inside the droplet.

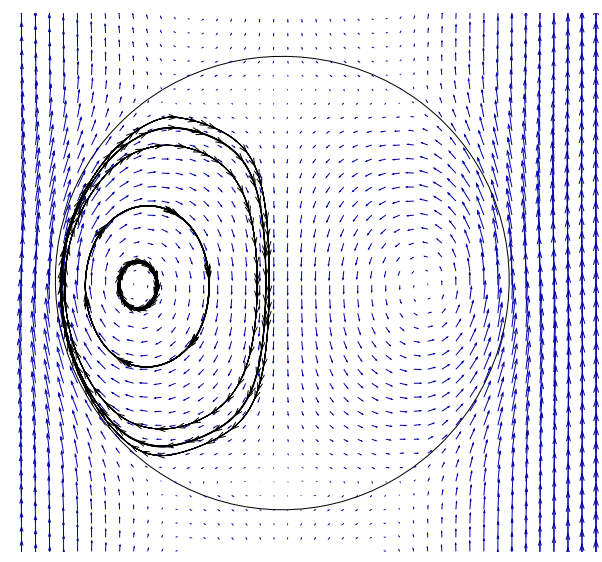

Fig. 9. Same unconfined microdroplet (black line) of Figure 8 together with the velocity field in the drop frame of reference (blue arrows) and some streamlines (in arrowed-line).

We then proposed a snapshot series of confined microdroplets. For all simu- 
lations, we used 36 cells to discretize the channel section. We checked that results are invariant under mesh refinement, which shows that computations are fully converged. We consider two sizes of droplets. One, which will be called the "small" droplet has a width of the order of the channel width $D$, namely $D=120$ micrometers and a length of order $1.3 D$. The other, which will be called the "big" droplet has a width of the order of the channel width $D$ and a length of order $2 D$. Injection velocity in the channel is $u_{i n}=0.2 \mathrm{~m} / \mathrm{s}$. Droplet viscosity is $2 \cdot 10^{-2} \mathrm{~kg} /(\mathrm{ms})$ and viscosity of the continuous fluid is $4.10^{-2} \mathrm{~kg} /(\mathrm{ms})$. Surface tension coefficient is $\sigma=3 \cdot 10^{-2} \mathrm{~N} / \mathrm{m}$. These data are referred to below as the "reference data". The small droplet is shown on Figure 10 with the velocity field and streamlines in the drop frame of reference, whereas the big droplet is shown on Figure 11.

Comparison of Figures 9, 10, 11 shows the modification of mixing dynamics induced by confinement and droplet volume. It appears that in every case there is a major mixing zone in the center of the droplet and two smaller zones, in the front and at the back of the droplet where the fluid is trapped. This has been observed both experimentally and numerically [10,37], by using passive tracers or dye inside the droplets. Figure 12 is a snapshot of a movie of physical experiment [10] of evolving microdroplets with passive tracers inside. The movie shows the motion of tracers along streamlines of the type of Figure 10 and also that some tracers are trapped at the back of the droplet, as it can be seen on Figure 12. Another physical experiment [10], emphasizing the presence of a zone in the front of the droplet which is not concerned by the flow at the center of the droplet (also referred as "dead zone" in the microdroplet community) is presented on Figure 13. In this experiment, dye is injected in the droplet and a chemical reaction occurs inside the droplet leading to a visual disappearance of the dye, when the droplets is transported along the channel (from left to right). We do not insist here on this reaction but on the mixing dynamics which can be observed inside the droplet. We see that dye does not propagate in the front of the droplet but is mainly carried along the central mixing zone (see the central droplet in Figure 13). This is an evidence of autonomous recirculation zone, in the front of the droplet, which does not exchange fluid with the center of the droplet.

Dynamics change due to viscosity switch between the two fluids is shown on Figure 14, which can be compared with Figure 10.

The effect of a change of injection velocity is shown on Figures 15 and 16 where injection velocity is set to $u_{i n}=0.1 \mathrm{~m} / \mathrm{s}$. Again droplet's shape is modified together with mixing dynamics inside. Increasing again the role of surface tension by lowering injection velocity to $u_{\text {in }}=0.05 \mathrm{~m} / \mathrm{s}$ has the effect shown on Figures 17 and 18. Comparing Figures 10/11, 15/16 and 17/18, we see that the increase of surface tension effect naturally induces more spherical interface at the front and the back of the droplet. 
The viscosity ratio between the droplet and the carrying fluid is also responsible for various mixing dynamics inside the droplet. Figures 19 and 20 show the results of simulations where viscosities are modified as follows : droplet viscosity is $2.10^{-3} \mathrm{~kg} /(\mathrm{ms})$ and viscosity of the continuous fluid is $2.10^{-2} \mathrm{~kg} /(\mathrm{ms})$, namely a viscosity ratio of 10 which arises in microfluidic applications. Comparing these figures with the references simulations, it appears that increase of viscosity ratio induces a strengthening of recirculation in the front and at the back of the droplet together with a backward motion of the core of central recirculation due to viscous coupling.

We also present a third droplet shape with width of the order of the channel width $D$ and a length of order $2.5 D$. We use reference data with two injection velocities $u_{\text {in }}=0.2 \mathrm{~m} / \mathrm{s}$ (Figure 21) and $u_{i n}=0.02 \mathrm{~m} / \mathrm{s}$ (Figure 22). We see that increasing influence of surface tension between Figures 21 and 22 leads to a more marked recirculation zone in the front of the droplet. This is shown by streamlines of Figure 22 : the second marked recirculation zone at the front of the droplet induces a decreased volume of the central mixing zone, compared to Figure 21.

Thus, using algorithms described in this paper, we can explore mixing dynamics inside microdroplets. This can help in the design of microflows configurations with microdroplets achieving the flow control needed in practical applications.

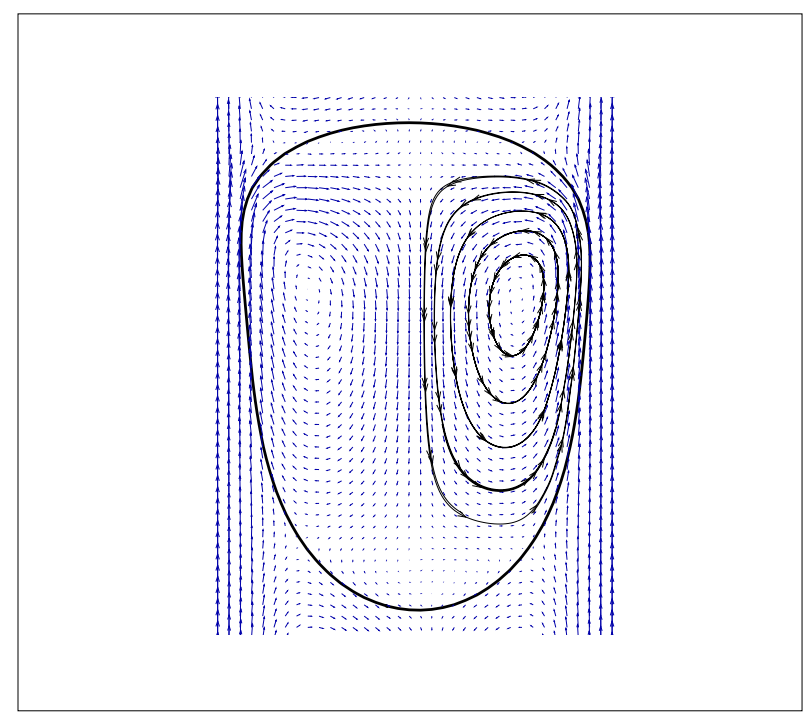

Fig. 10. Small droplet (black line) with the velocity field in the drop frame of reference (blue arrows) and some streamlines (in arrowed-line) ; reference data. 


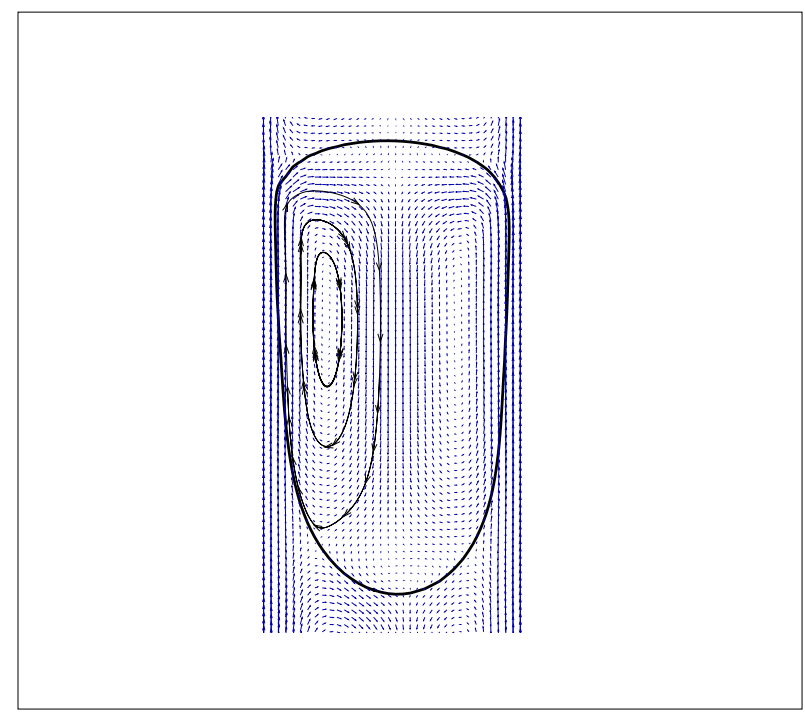

Fig. 11. Big droplet (black line) with the velocity field in the drop frame of reference (blue arrows) and some streamlines (in arrowed-line); reference data. 


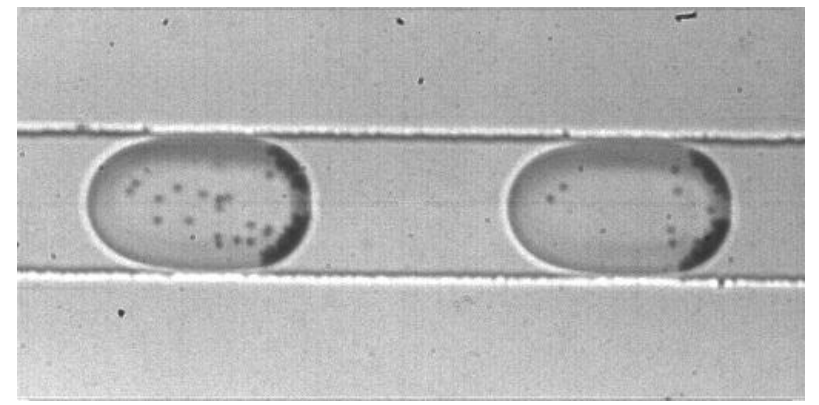

Fig. 12. A snapshot of a physical experiment [10] : two moving microdroplets (from right to left) with passive tracers inside (small dark points) ; the movie from which is extracted this photograph shows that some tracers moves along streamlines of the type of Figure 10 and also that some tracers are trapped at the back of the droplet.

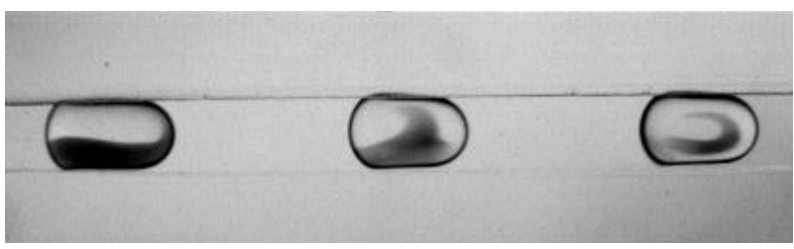

Fig. 13. Another snapshot of a physical experiment [10] : three moving microdroplets (from left to right) with dye inside (dark color) ; the movie from which is extracted this photograph shows that dye is mainly carried along the central mixing zone and does not propagate in the front of the droplet.

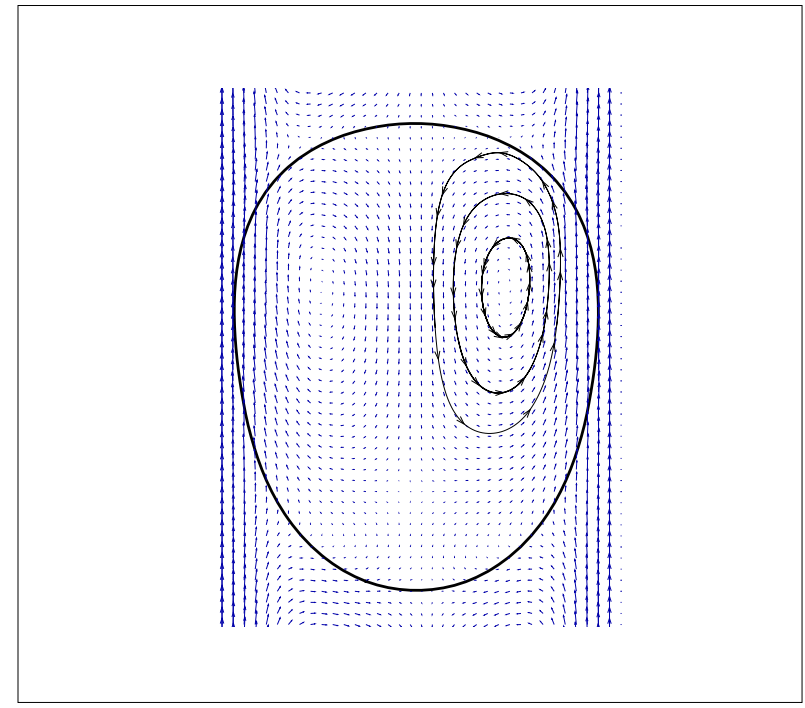

Fig. 14. Small droplet (black line) with the velocity field in the drop frame of reference (blue arrows) and some streamlines (in arrowed-line) ; reference data except that viscosities inside and outside the droplet are switched 


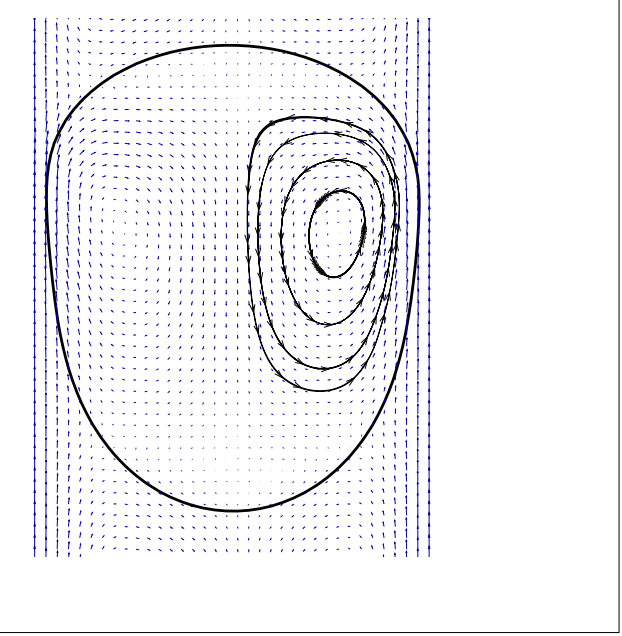

Fig. 15. Small droplet (black line) with the velocity field in the drop frame of reference (blue arrows) and some streamlines (in arrowed-line) ; reference data but for $u_{\text {in }}=0.1 \mathrm{~m} / \mathrm{s}$

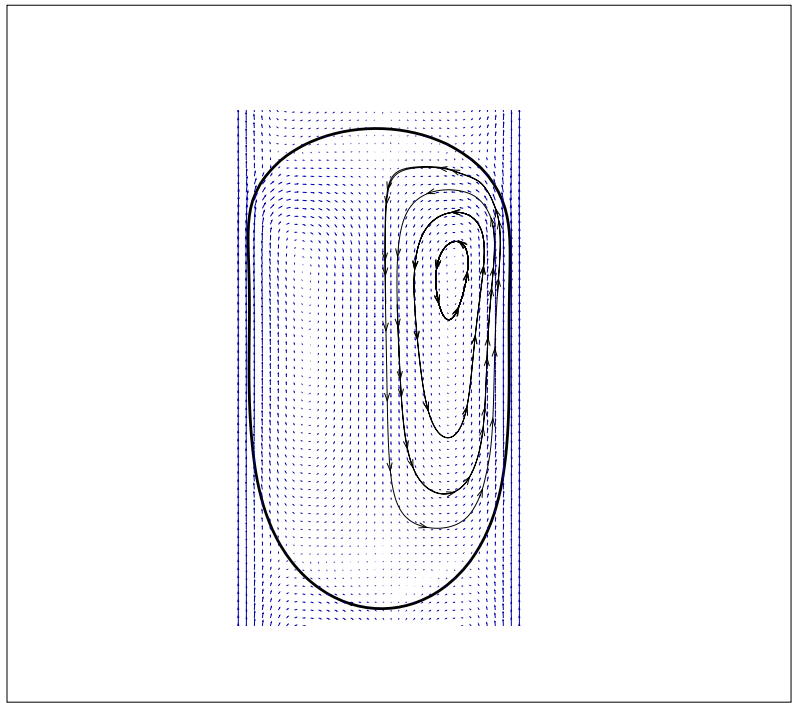

Fig. 16. Big droplet (black line) with the velocity field in the drop frame of reference (blue arrows) and some streamlines (in arrowed-line) ; reference data but for $u_{\text {in }}=$ $0.1 \mathrm{~m} / \mathrm{s}$ 


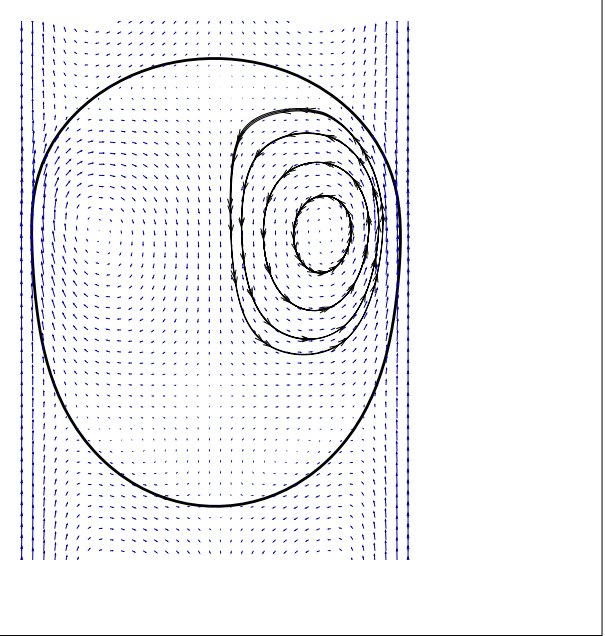

Fig. 17. Small droplet (black line) with the velocity field in the drop frame of reference (blue arrows) and some streamlines (in arrowed-line) ; reference data but for $u_{i n}=0.05 \mathrm{~m} / \mathrm{s}$

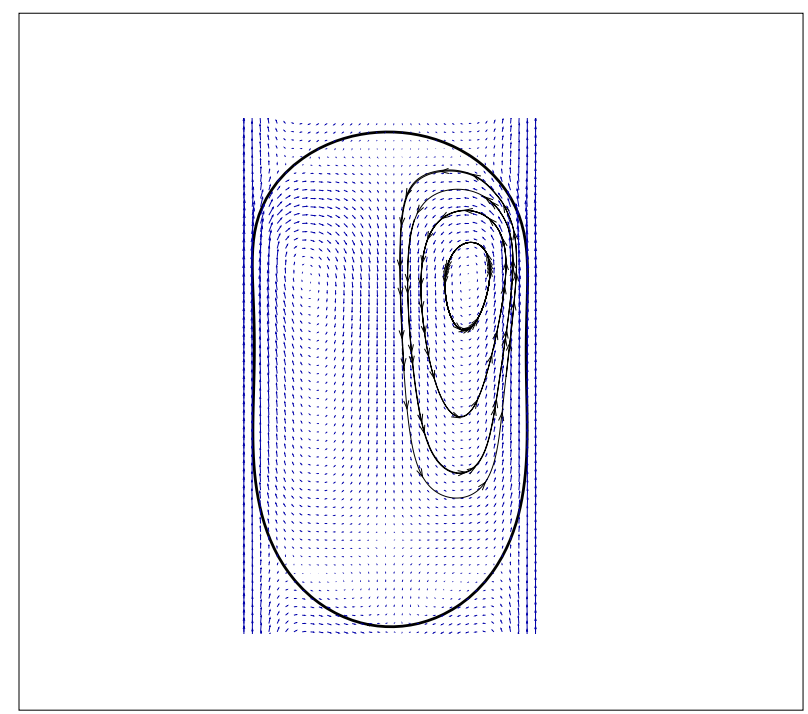

Fig. 18. Big droplet (black line) with the velocity field in the drop frame of reference (blue arrows) and some streamlines (in arrowed-line) ; reference data but for $u_{\text {in }}=$ $0.05 \mathrm{~m} / \mathrm{s}$ 


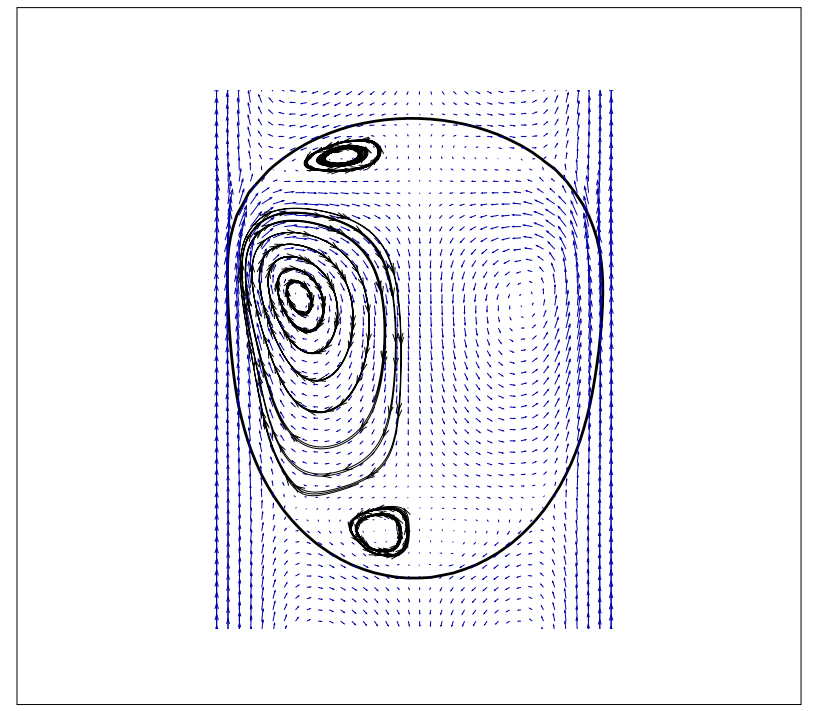

Fig. 19. Small droplet (black line) with the velocity field in the drop frame of reference (blue arrows) and some streamlines (in arrowed-line) ; reference data but for viscosity ratio (see text)

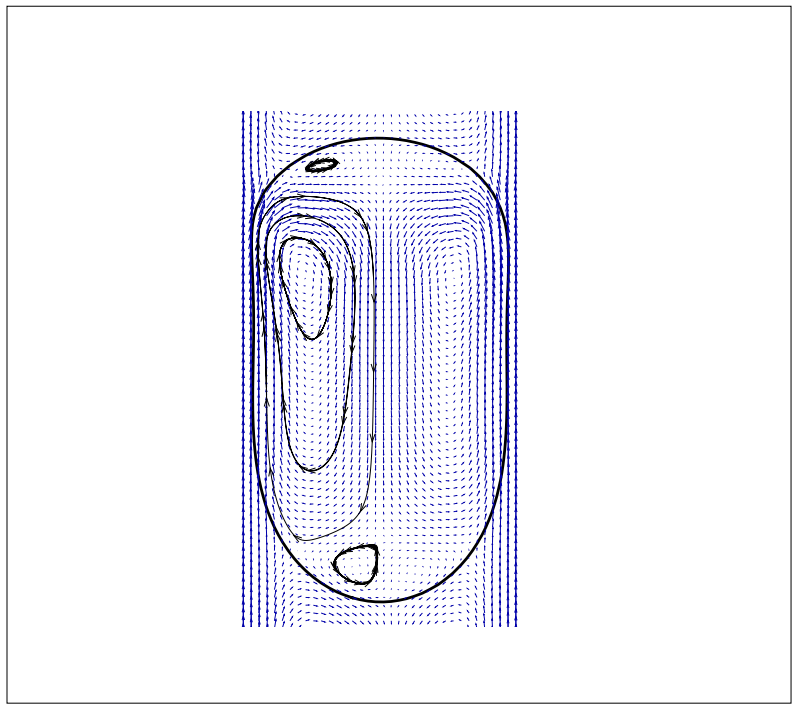

Fig. 20. Big droplet (black line) with the velocity field in the drop frame of reference (blue arrows) and some streamlines (in arrowed-line) ; reference data but for viscosity ratio (see text) 


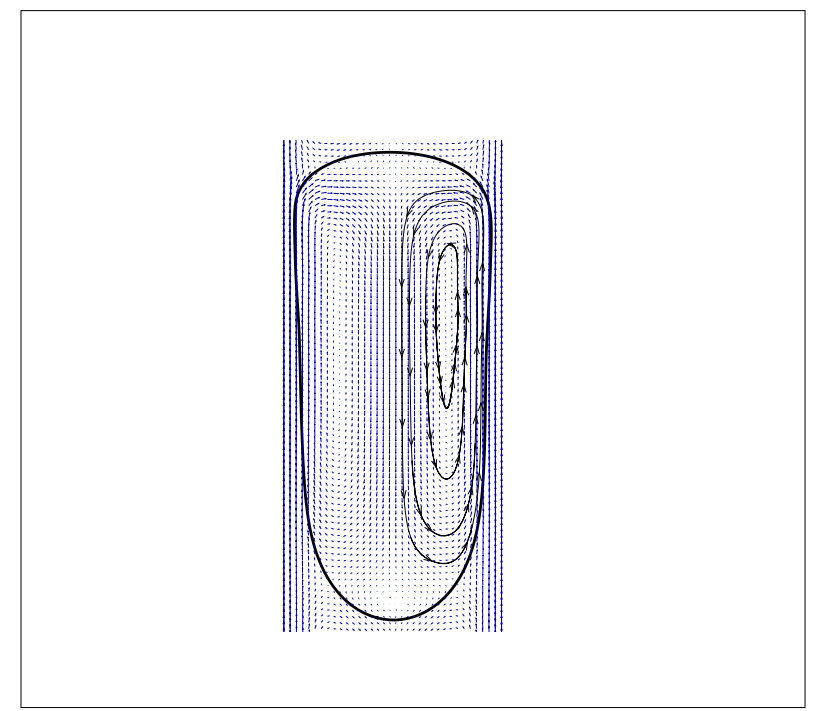

Fig. 21. Big droplet (black line) with the velocity field in the drop frame of reference (blue arrows) and some streamlines (in arrowed-line) ; reference data but for droplet's length of $2.5 \mathrm{D}$

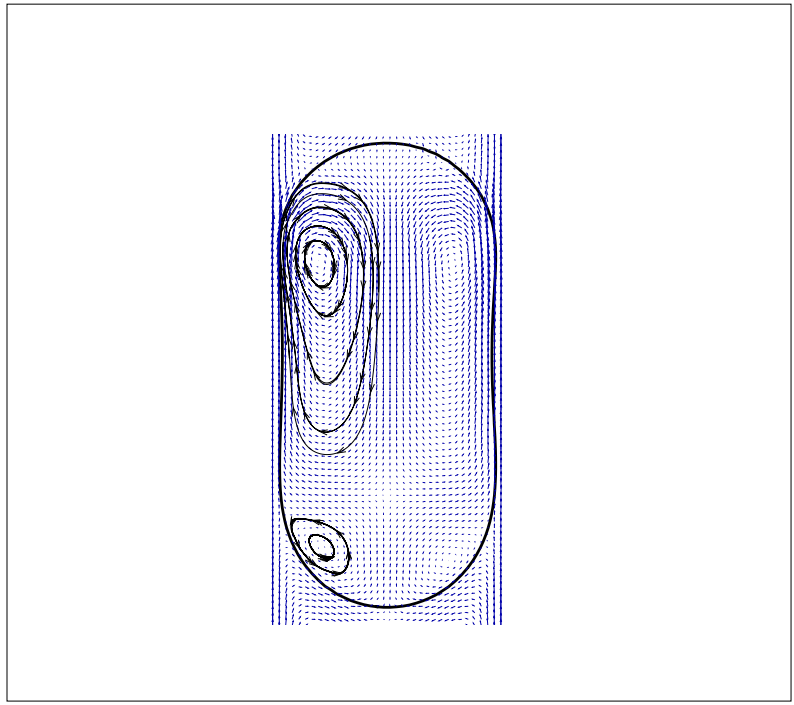

Fig. 22. Big droplet (black line) with the velocity field in the drop frame of reference (blue arrows) and some streamlines (in arrowed-line) ; reference data but for droplet's length of $2.5 D$ and $u_{i n}=0.02 \mathrm{~m} / \mathrm{s}$ 


\section{Conclusion}

In this paper, we derived a new stability condition induced by the explicit discretization of the surface tension term in incompressible bifluid models adopting CSF method. This new stability condition is adapted for the whole Reynolds numbers and degenerates to the stability condition of Brackbill et al. for low viscosities (leading to high Reynolds numbers). Moreover, in the case of low densities or small domain (leading to low Reynolds), our stability condition degenerates to a condition which involves viscosity instead of density and is better suited for such flows. This latter condition was validated by a numerical study and if it is transgressed, parasitic currents can occur near the interface. To sum up it appears this general stability condition is practically equivalent to take the maximum of these two previous time steps : this maximum allows to pick up the appropriate condition with respect to the associated regime (high or low Reynolds numbers). A numerical code was developed based on essential tools of the Level Set technology, namely WENO5 schemes, TVD Runge-Kutta schemes and PDE based redistanciation were used to achieve accurate simulations of surface tension-driven flows. In a staggered grid framework, we used an augmented Lagrangian method to solve incompressible Navier-Stokes equation.

We validated the new stability condition by simulating microflows and exploring various mixing dynamics inside microdroplets. This is the first such demonstration we are aware of in the context of numerical simulation of moving microdroplets in straight microchannels, where mixing dynamics dependence on confinement, droplet volume, injection velocity and viscosity ratio is studied. Our numerical results are in good agreement with physical experiments and available results of the literature.

Algorithms developed in this paper allow for simulations which can help in the design of microflows configurations with microdroplets achieving the flow control needed in practical applications. In future work, we will study $3 \mathrm{D}$ effects on microdroplets dynamics with a 3D code.

\section{Acknowledgements}

This work was partially supported by ACI-NIM "Calculs de micro-fluides" (CNRS) and ANR-SCAN2. We also warmly thank Annie Colin and Galder Cristobal, from the Rhodia-LOF laboratory (Pessac, France), for providing us results of physical experiments with microdroplets and fruitful discussions about this work. 


\section{A Velocity in the drop frame of reference}

In microfluidics, due to surface tension, interfaces converge quickly towards stationary shape. In this section, we describe a method for microflows in straight channels which makes the most of the stationary shapes of the interfaces. By working in the drop frame of reference, the normal velocity vanishes at droplet shape equilibrium. Small normal velocity is the criterion of stabilized asymptotic shape. Then, in a straight channel, the global flow is the superposition of the velocity in the droplet frame of reference and the constant translation velocity of the droplet.

To compute such a decomposition, we thus need to define this latter scalar droplet velocity. This velocity has a meaning as soon as the shape of the droplet is stabilized and then there exist a scalar $u_{d}$ such that

$$
\mathbf{u}=u_{d} \mathbf{U}+\mathbf{v}, \mathbf{v} \cdot \mathbf{n}=0 \text { on } \Gamma_{0},
$$

where $\mathbf{U}$ is a "unitary" flow parallel to the wall in a regular channel. Then the droplet moves with the global velocity $u_{d} \mathbf{U}$ and the velocity in the drop frame of reference is $\mathbf{v}$.

In the general case, with stabilized or destabilized droplet shape, we define the local droplet's velocity on the fluid interface where $\mathbf{U} \cdot \mathbf{n} \neq 0$ as

$$
u_{d}^{l o c}=\frac{\mathbf{u} \cdot \mathbf{n}}{\mathbf{U} \cdot \mathbf{n}} \text { on } \Gamma_{0} .
$$

We then define the droplet's velocity $u_{d}$ as the mean value of local droplet velocities, where $\mathbf{U} \cdot \mathbf{n}$ is far from zero. When the local droplet velocities are close to be identical along $\Gamma_{0}$, the normal global velocity, $\mathbf{u} \cdot \mathbf{n}$, verifies, by virtue of definition (A.2),

$$
\mathbf{u} \cdot \mathbf{n}=u_{d} \mathbf{U} \cdot \mathbf{n} \text { on } \Gamma_{0}
$$

It follows that definition (A.1) is fulfilled, in particular, $\mathbf{v} \cdot \mathbf{n}=0$ on $\Gamma_{0}$. We are then concerned with a stabilized interface $\Gamma_{0}$ moving with the scalar velocity $u_{d}$ along the channel direction. Criteria of a stabilized interface are the smallness of quantity $\mathbf{v} \cdot \mathbf{n}$ or (and it is equivalent) uniform local velocities in the sense of (A.2). In our code, we use this latter criterion in order to avoid useless iterations.

There are several advantages in determining $u_{d}$. First, knowing $u_{d}$ allows to plot the velocity in the droplet frame of reference $\mathbf{v}$ and to analyse the mixing dynamics inside the droplet. Second, when the goal is only to analyse the 
velocity field in the drop frame of reference for a simple channel, it is not necessary to follow the displacement of the droplet. We then transport the interface only with velocity $\mathbf{v}$ instead of $\bar{u}_{d} \mathbf{U}+\mathbf{v}$ and we can use a small channel containing the droplet to reduce the numerical cost. As a matter of fact, by working in the drop frame of reference, the droplet does not translate and its shape converges to the stabilized shape. Third, this static asymptotic allows a better convergence of the droplet's shape and velocity field. In particular, the computations of viscosity and curvature do not change asymptotically on the grid. 


\section{References}

[1] D. M. Anderson, G. B. McFadden, and A. A. Wheeler. Diffuse-interface methods in fluid mechanics. Annual Review of Fluid Mechanics, 30(1):139-165, 1998.

[2] S. L. Anna, N. Bontoux, and H. A. Stone. Formation of dispersions using "flow focusing" in microchannels. Applied Physics Letters, 82(3):364-366, 2003.

[3] E. Bänsch. Finite element discretization of the Navier-Stokes equations with a free capillary surface. Numer. Math., 88(2):203-235, 2001.

[4] F. Boyer. A theoretical and numerical model for the study of incompressible mixture flows. Computers \& Fluids, 31(1):41-68, January 2002.

[5] J. U. Brackbill, D. B. Kothe, and C. Zemach. A continuum method for modeling surface tension. Journal of Computational Physics, 100(2):335-354, 1992.

[6] Y. C. Chang, T. Y. Hou, B. Merriman, and S. Osher. A level set formulation of eulerian interface capturing methods for incompressible fluid flows. Journal of Computational Physics, 124(2):449-464, March 1996.

[7] S. Chen and G. D. Doolen. Lattice boltzmann method for fluid flows. Annual Review of Fluid Mechanics, 30(1):329-364, 1998.

[8] D. L. Chopp. Computing minimal surfaces via level set curvature flow. J. Comput. Phys, 106:77-91, 1993.

[9] A. J. Chorin. A numerical method for solving incompressible viscous flow problems. J. Comput. Phys., 2(1):12-26, 1967.

[10] A. Colin and G. Cristobal. private communication, 2006.

[11] M. Fortin and R. Glowinsky. Augmented Lagrangian methods : applications to the numerical solution of boundary-value problems, volume 15 of Series in Mathematics and its applications. North-Holland, 1983.

[12] I. Ginzburg and K. Steiner. Lattice boltzmann model for free-surface flow and its application to filling process in casting. J. Comput. Phys., 185(1):61-99, 2003.

[13] F. H. Harlow and J. E. Welch. Numerical calculation of time-dependent viscous incompressible flow of fluid with free surface. Physics of Fluids, 8(12):21822189, 1965.

[14] C. Hirt and B. Nichols. Volume of fluid (VOF) method for the dynamics of free boundaries. J. Comput. Phys., 39:201-225, 1981.

[15] S. Hysing. A new implicit surface tension implementation for interfacial flows. International Journal for Numerical Methods in Fluids, 51(6):659-672, 2006.

[16] T. Inamuro, T. Ogata, S. Tajima, and N. Konishi. A lattice boltzmann method for incompressible two-phase flows with large density differences. J. Comput. Phys., 198(2):628-644, 2004. 
[17] D. Jamet, O. Lebaigue, N. Coutris, and J. M. Delhaye. The second gradient method for the direct numerical simulation of liquid-vapor flows with phase change. J. Comput. Phys., 169(1):624-651, 2001.

[18] D. Jamet, D. Torres, and J. U. Brackbill. On the theory and computation of surface tension: the elimination of parasitic currents through energy conservation in the second-gradient method. J. Comput. Phys., 182(1):262$276,2002$.

[19] G.-S. Jiang and D. Peng. Weighted eno schemes for hamilton-jacobi equations. SIAM Journal of Scientific Computing, 21(No. 6):2126-2143, 2000.

[20] G.-S. Jiang and C.-W. Shu. Efficient implementation of weighted eno schemes. J. Comput. Phys, 126:202-228, 1996.

[21] M. Joanicot and A. Ajdari. APPLIED PHYSICS: Droplet Control for Microfluidics. Science, 309(5736):887-888, 2005.

[22] M. Kang, R. P. Fedkiw, and X.-D. Liu. A boundary condition capturing method for multiphase incompressible flow. J. Sci. Comput., 15(No. 3):323-360, 2000.

[23] J. Kim. A continuous surface tension force formulation for diffuse-interface models. J. Comput. Phys., 204(2):784-804, 2005.

[24] B. Lafaurie, C. Nardone, R. Scardovelli, S. Zaleski, and G. Zanetti. Modelling merging and fragmentation in multiphase flows with surfer. J. Comput. Phys., 113(1):134-147, 1994.

[25] A. Malidi, S. Dufour, and D. N'dri. A study of time integration schemes for the numerical modelling of free surface flows. International Journal for Numerical Methods in Fluids, 48(10):1123-1147, 2005.

[26] E. Marchandise, P. Geuzaine, N. Chevaugeon, and J.-F. Remacle. A stabilized finite element method using a discontinuous level set approach for the computation of bubble dynamics. J. Comput. Phys., 2007.

[27] M. D. Menech. Modeling of droplet breakup in a microfluidic t-shaped junction with a phase-field model. Physical Review E (Statistical, Nonlinear, and Soft Matter Physics), 73(3):031505, 2006.

[28] W. Mulder, S. Osher, and J. Sethian. Computing interface motion in compressible gas dynamics. J. Comput. Phys, 100:209-228, 1992.

[29] E. Olsson and G. Kreiss. A conservative level set method for two phase flow. J. Comput. Phys., 210(1):225-246, 2005.

[30] S. Osher and R. Fedkiw. Level Set Methods and Dynamic Implicit Surfaces, volume 153 of Applied Mathematical Sciences. Springer, 2003.

[31] S. Osher and J. Sethian. Fronts propagating with curvature dependant speed : Algorithms based on hamilton-jacobi formulations. J. Comput. Phys, 79:12-49, 1988. 
[32] S. Popinet and S. Zaleski. A front-tracking algorithm for accurate representation of surface tension. International Journal for Numerical Methods in Fluids, 30(6):775-793, 1999.

[33] Y. Renardy. The effects of confinement and inertia on the production of droplets. Rheologica Acta, 46(4):521-529, 2007.

[34] Y. Renardy and M. Renardy. Prost: a parabolic reconstruction of surface tension for the volume-of-fluid method. J. Comput. Phys., 183(2):400-421, 2002.

[35] M. Rudman. A volume-tracking method for incompressible multifluid flows with large density variations. International Journal for Numerical Methods in Fluids, 28(2):357-378, 1998.

[36] G. Russo and P. Smereka. A remark on computing distance functions. J. Comput. Phys., 163(1):51-67, 2000.

[37] F. Sarrazin, K. Loubière, L. Prat, C. Gourdon, T. Bonometti, and J. Magnaudet. Experimental and numerical study of droplets hydrodynamics in microchannels. AIChE Journal, 52(12):4061-4070, 2006.

[38] R. Scardovelli and S. Zaleski. Direct numerical simulation of free-surface and interfacial flow. Annu. Rev. Fluid Mech., (31):567-603, 1999.

[39] J. A. Sethian. Level Set Methods and Fast Marching Methods - Evolving interfaces in computational geometry, fluid mechanics, computer vision and materials science, volume 3 of Cambridge Monographs on Applied and Computational Mathematics. Cambridge University Press, second edition, 1999.

[40] E. Shapiro and D. Drikakis. Artificial compressibility, characteristicsbased schemes for variable-density, incompressible, multispecies flows: part ii. multigrid implementation and numerical test. J. Comput. Phys., 210(2):608$631,2005$.

[41] S. Shin, S. I. Abdel-Khalik, V. Daru, and D. Juric. Accurate representation of surface tension using the level contour reconstruction method. J. Comput. Phys., 203(2):493-516, 2005.

[42] E. Shirani, N. Ashgriz, and J. Mostaghimi. Interface pressure calculation based on conservation of momentum for front capturing methods. J. Comput. Phys., 203(1):154-175, 2005.

[43] C.-W. Shu and S. Osher. Efficient implementation of essentially non-oscillatory shock-capturing schemes. J. Comput. Phys, 77:439-471, 1988.

[44] C.-W. Shu and S. Osher. Efficient implementation of essentially non-oscillatory shock-capturing schemes II. J. Comput. Phys, 83:32-78, 1989.

[45] H. Song and R. Ismagilov. Millisecond kinetics on a microfluidic chip using nanoliters of reagents. Journal of the American Chemical Society, 125(47):14613-14619, 2003. 
[46] H. Stone, A. Stroock, and A. Ajdari. Engineering flows in small devices: Microfluidics toward a lab-on-a-chip. Annual Review of Fluid Mechanics, 36:381-411, 2004.

[47] M. Sussman, A. S. Almgren, J. B. Bell, P. Colella, L. H. Howell, and M. L. Welcome. An adaptive level set approach for incompressible two-phase flows. J. Comput. Phys., 148(1):81-124, 1999.

[48] M. Sussman, E. Fatemi, P. Smereka, and S. Osher. An improved level set method for incompressible two-phase flows. Computers \& Fluids, 27:663-680, 1998.

[49] M. Sussman, P. Smereka, and S. Osher. A level set approach for computing solutions to incompressible two-phase flow. J. Comput. Phys, 114:146-159, 1994.

[50] R. Temam. Sur l'approximation des solutions des équations de Navier-Stokes. C. R. Acad. Sci. Paris Sér. A, 262:219-221, 1966.

[51] R. Temam. Navier-Stokes equations. Theory and numerical analysis. NorthHolland, 1977.

[52] A. Y. Tong and Z. Wang. A numerical method for capillarity-dominant free surface flows. Journal of Computational Physics, 221(2):506-523, February 2007.

[53] D. J. Torres and J. U. Brackbill. The point-set method: front-tracking without connectivity. J. Comput. Phys., 165(2):620-644, 2000.

[54] G. Tryggvason, B. Bunner, A. Esmaeeli, D. Juric, N. Al-Rawahi, W. Tauber, J. Han, S. Nas, and Y.-J. Jan. A front-tracking method for the computations of multiphase flow. J. Comput. Phys., 169(2):708-759, 2001.

[55] S. O. Unverdi and G. Tryggvason. A front-tracking method for viscous, incompressible, multi-fluid flows. J. Comput. Phys., 100(1):25-37, 1992.

[56] J.-D. Yu, S. Sakai, and J. Sethian. A coupled level set projection method applied to ink jet simulation. Interfaces and Free Boundaries, 5(4):459-482, 2003.

[57] J.-D. Yu, S. Sakai, and J. Sethian. A coupled quadrilateral grid level set projection method applied to ink jet simulation. Journal of Computational Physics, 206(1):227-251, June 2005.

[58] J.-D. Yu, S. Sakai, and J. Sethian. Two-phase viscoelastic jetting. Journal of Computational Physics, 220(2):568-585, January 2007. 